\title{
Model-Reference Adaptive Control of Distributed Lagrangian Infinite-Dimensional Systems Using Hamilton's Principle
}

\author{
Nhan Nguyen* \\ Kelley Hashemi ${ }^{\dagger}$ \\ NASA Ames Research Center, Moffett Field, CA 94035 \\ Ehsan Arabi ${ }^{\ddagger}$ \\ University of Michigan, Ann Arbor, MI 48109 \\ Tansel Yucelen ${ }^{\S}$ \\ University of South Florida, Tampa, FL 33620
}

\begin{abstract}
This paper presents a Hamilton's principle for distributed control of infinite-dimensional systems modeled by a distributed form of the Euler-Lagrange method. The distributed systems are governed by a system of linear partial differential equations in space and time. A generalized potential energy expression is developed that can capture most physical systems including those systems that have no spatial distribution. The Hamilton's principle is applied to derive distributed feedback control methods without resorting to the standard weak-form discretization approach to convert an infinite-dimensional systems to a finite-dimensional systems. It can be shown by the principle of least action that the distributed control synthesized by the Hamilton's principle is a minimum-norm control. A model-reference adaptive control framework is developed for distributed Lagrangian systems in the presence of uncertainty. The theory is demonstrated by an application of adaptive flutter suppression control of a flexible aircraft wing.
\end{abstract}

\section{Introduction}

In control theory, infinite dimensional systems are systems that have distributions over both spatial and temporal dimensions. Such systems are highly common in many physical applications and are usually referred to as distributedparameter systems or simply distributed systems. In fact, most physical systems that exist in the physical world are distributed in nature. A common system in aerospace engineering is an aircraft which is actually a distributedparameter system wherein the behavior of every point on the aircraft is governed by distributions of aerodynamic pressure forces, elastic forces, and body forces, as well as the structural and mass properties.

Control theories of distributed systems can address the spatial distribution of physical parameters of those systems, but in the majority of control applications, this is not the preferred approach due to the complicated nature of the spatial distribution in those theories. Moreover, the state information for distributed systems cannot be measured in its entirety due to the infinite-dimensionality of the system state variables. Therefore, model reduction and spatial discretization techniques are normally employed to reduce a distributed-parameter infinite-dimensional system to a lumped-parameter finite-dimensional system. A typical lumped-parameter 6-degree-of-freedom aircraft flight dynamic model is obtained through a model reduction technique by integrating the aerodynamic pressure forces over the spatial dimension of the aircraft. Distributed-parameter systems are generally modeled by partial differential equations (PDE), whereas lumped-parameter systems are usually modeled by ordinary differential equations (ODE).

\footnotetext{
${ }^{*}$ Senior Research Scientist and Technical Group Lead of Advanced Control and Evolvable Systems Group, Intelligent Systems Division, nhan.t.nguyen@nasa.gov

${ }^{\dagger}$ Research Scientist, Intelligent Systems Division, kelley.e.hashemi@nasa.gov

${ }^{\ddagger}$ Postdoctoral Research Fellow, Aerospace Engineering Department, earabi@umich.edu

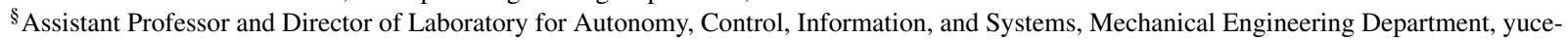
len@usf.edu
} 
In structural dynamic applications, a standard spatial discretization technique such as the Galerkin's or finiteelement method is normally used to reduce a distributed-parameter system to a finite-dimensional system using an integral weak-form approximation of the strong-form partial differential equation. ${ }^{1}$ The order of the approximate finite-dimensional systems must be sufficiently high in order to preserve the accuracy of the model. As the order of a finite-dimensional system increases, control of the system becomes more complex. Furthermore, the state information of the finite-dimensional system usually is unavailable for a full-state feedback control synthesis. Using outputs from sensors, an output feedback control or linear-quadratic Gaussian (LQG) control can be synthesized from the finitedimensional system. ${ }^{9}$

A typical approach in control of finite-dimensional systems with large number of states is model reduction which condenses a high-order finite-dimensional model into a simpler lower-order model that can capture most of the important physical effects. Balanced realization is a common model reduction technique frequently used in control applications. ${ }^{6,7}$ The reduced-order model is then used in a control synthesis. While the model reduction approach is well understood, the possibility of introducing model reduction errors may exist in a control design as a consequence of the elimination of extra state variables from the original high-order finite-dimensional system. Therefore, the control design must ensure high degree of robustness in safeguarding the potential introduction of improper control actions into the system as a result of possible model reduction errors.

An equivalent control design philosophy for infinite-dimensional systems could be employed. A control design can be synthesized for an infinite-dimensional system without approximating the system using a finite-dimensional representation. Once a control design has been developed, the control design is approximated by spatial discretization to transform the distributed state information contained in the control design into the output information based on sensor measurements.

The lack of popularity of distributed-parameter system control applications is perhaps due to the lack of distributed sensing capabilities that can measure spatially distributed state information. Biological systems may have these distributed sensing capabilities such as birds which can sense pressure distribution over their entire bodies. For engineering systems, distributed sensing capabilities are still limited and thus practical applications of control theory for distributed systems are not commonly found. In recent years, some distributed sensing capabilities have been developed for aircraft structures such as the fiber-optic strain and shape sensing (FOSS) technology. ${ }^{5}$ With this sensing technology, practical applications of distributed system control theory could see renewed interests.

Most physical distributed systems are governed by the energy principle of thermodynamics. Motions of dynamical systems can be characterized by the kinetic energy and potential energy. Lagrangian mechanics is the natural setting for analyzing dynamical systems using the energy approach. ${ }^{8}$ The Hamilton's principle in classical dynamics is connected to the notion of energy conservation for a conservative system. ${ }^{8}$ The Hamiltonian function represents the total energy of a system. By examining the changes of the Hamiltonian function, stability of a system can be assessed. The Hamiltonian function is a positive-definite quantity much like a Lyapunov function. In fact, the Hamiltonian function could be thought of as a special case of a Lyapunov function. For a mechanical system, finding a Lyapunov function can be difficult, but every mechanical system possesses a Hamiltonian function which could be constructed.

In this paper, we develop a general method for a class of distributed systems with spatial distribution using the Lagrangian mechanics. Using the Hamilton's principle, a number of distributed control methods can be synthesized as a feedback of the spatially distributed state information which is assumed to be available from distributed sensor technologies such as FOSS. The distributed controls are obtained in integral strong-form expressions without approximation by spatial discretization. It can be shown by the principle of least action that the distributed control synthesized by the Hamilton's principle is a minimum-norm control. A model-reference adaptive control framework is developed based on the Hamilton's principle for this class of distributed systems in the presence of uncertainty.

The distributed control theory is then demonstrated by an application of adaptive flutter suppression control of a flexible aircraft wing without using the standard output feedback or LQG approach for the finite-dimensional representation of the aeroelastic equations of motion of the flexible wing.

\section{Distributed Lagrangrian Systems}

Consider a distributed system (S) modeled by an initial-boundary value problem

$$
m(x) \frac{\partial^{2} w}{\partial t^{2}}-f\left(x, w, \frac{\partial w}{\partial x}, \ldots \frac{\partial^{m} w}{\partial x^{m}}\right)=q\left(x, t, w, \frac{\partial w}{\partial x}, \ldots, \frac{\partial^{m-1} w}{\partial x^{m-1}}, \frac{\partial w}{\partial t}, u\right)
$$


expressed in a strong form whose solution $w(x, t): \Omega \times[0, \infty) \rightarrow \mathbb{R}^{n}$ is defined over an open bounded domain $x \in \Omega=$ $(a, b) \subset \mathbb{R}$ with suitable boundary conditions on the boundary $\partial \Omega$ for all $t \in[0, \infty)$. The initial values are specified by $w(x, 0)=w_{0}(x)$ and $\frac{\partial w(x, 0)}{\partial t}=w_{t_{0}}(x)$. The strong-form solution $w(x, t) \in C_{c}^{\infty}$ is assumed to be continuous and infinitely differentiable. The distributed control $u(x, t)$ is spatially distributed over a bounded subspace $x \in \Omega_{c}=$ $\left(a_{c}, b_{c}\right) \subset \Omega \subset \mathbb{R}$.

The function $m(x)>0 \in \mathbb{R}^{n} \times \mathbb{R}^{n}$ is a positive-valued function distributed over $x$. The function $f\left(x, w, \frac{\partial w}{\partial x}, \ldots \frac{\partial^{m} w}{\partial x^{m}}\right) \in$ $\mathbb{R}^{n}$ depends only on the spatial partial derivatives of the solution $w(x, t)$. The function $q\left(x, w, \frac{\partial w}{\partial x}, \frac{\partial^{m-1} w}{\partial x^{m-1}} \frac{\partial w}{\partial t}\right) \in \mathbb{R}^{n}$ is a non-homogeneous term that depends on both spatial and temporal partial derivatives.

We analyze the distributed system (S) using the principle of virtual work. Toward that end, we introduce the virtual function $\delta w(x, t)$. We now state the following lemma:

Lemma 1: If there exists a scalar positive-valued function $v\left(x, w, \frac{\partial w}{\partial x}, \ldots \frac{\partial^{m} w}{\partial x^{m}}\right)>0 \in \mathbb{R}$ such that

$$
\delta w \cdot f=-\delta w \cdot \sum_{i=0}^{m}(-1)^{i} \frac{\partial^{i}}{\partial x^{i}}\left(\frac{\partial v}{\partial\left(\frac{\partial^{i} w}{\partial x^{i}}\right)}\right)=-\delta w \cdot\left[\frac{\partial v}{\partial w}-\frac{\partial}{\partial x}\left(\frac{\partial v}{\partial\left(\frac{\partial w}{\partial x}\right)}\right)+\cdots+(-1)^{m} \frac{\partial^{m}}{\partial x^{m}}\left(\frac{\partial v}{\partial\left(\frac{\partial^{m} w}{\partial x^{m}}\right)}\right)\right]
$$

where the $\cdot$ symbol denotes a scalar product operation of two vectors, and

$$
\delta \phi_{i j}(x)=\left(\frac{\partial^{j-i-1} \delta w}{\partial x^{j-i-1}}\right) \cdot \frac{\partial^{i}}{\partial x^{i}}\left(\frac{\partial v}{\partial\left(\frac{\partial j_{w}}{\partial x^{j}}\right)}\right)
$$

has compact support in $\Omega$ with vanishing boundary values on $\partial \Omega^{2}$ for all $0 \leq i \leq m-1$ and $1 \leq j \leq m$, then $v\left(x, w, \frac{\partial w}{\partial x}, \ldots \frac{\partial^{m} w}{\partial x^{m}}\right)$ is called a potential energy density and $f\left(x, w, \frac{\partial w}{\partial x}, \ldots \frac{\partial^{m} w}{\partial x^{m}}\right)$ is called a conservative distributed force.

Proof: Consider the virtual work done by $f\left(x, w, \frac{\partial w}{\partial x}, \ldots \frac{\partial^{m} w}{\partial x^{m}}\right)$ which is expressed as

$$
\delta W=\int_{\Omega} \delta w \cdot f d x=-\int_{\Omega} \delta v d x=-\int_{\Omega}\left[\delta w \cdot \frac{\partial v}{\partial w}+\delta\left(\frac{\partial w}{\partial x}\right) \cdot \frac{\partial v}{\partial\left(\frac{\partial w}{\partial x}\right)}+\cdots+\delta\left(\frac{\partial^{m} w}{\partial x^{m}}\right) \cdot \frac{\partial v}{\partial\left(\frac{\partial^{m_{w}}}{\partial x^{m}}\right)}\right] d x
$$

Upon successive integration by parts, we obtain

$$
\begin{gathered}
\int_{\Omega} \delta w \cdot f d x=-\int_{\Omega} \delta w \cdot\left[\frac{\partial v}{\partial w}-\frac{\partial}{\partial x}\left(\frac{\partial v}{\partial\left(\frac{\partial w}{\partial x}\right)}\right)+\cdots+(-1)^{m} \frac{\partial^{m}}{\partial x^{m}}\left(\frac{\partial v}{\partial\left(\frac{\partial^{m} w}{\partial x^{m}}\right)}\right)\right] \\
-\left[\delta w \cdot \frac{\partial v}{\partial\left(\frac{\partial w}{\partial x}\right)}-\cdots+\delta\left(\frac{\partial^{m-1} w}{\partial x^{m-1}}\right) \cdot \frac{\partial v}{\partial\left(\frac{\partial^{m} w}{\partial x^{m}}\right)}-\delta\left(\frac{\partial^{m-2} w}{\partial x^{m-2}}\right) \cdot \frac{\partial}{\partial x}\left(\frac{\partial v}{\partial\left(\frac{\partial^{m} w}{\partial x^{m}}\right)}\right)\right. \\
\left.+\cdots+\delta w \cdot(-1)^{m-1} \frac{\partial^{m-1}}{\partial x^{m-1}}\left(\frac{\partial v}{\partial\left(\frac{\partial^{m} w}{\partial x^{m}}\right)}\right)\right]_{\partial \Omega} \\
=-\int_{\Omega} \delta w \cdot \sum_{i=0}^{m}(-1)^{i} \frac{\partial^{i}}{\partial x^{i}}\left(\frac{\partial v}{\partial\left(\frac{\partial^{i} w}{\partial x^{i}}\right)}\right) d x-\left.\sum_{j=1}^{m} \sum_{i=0}^{j-1}(-1)^{i} \delta \phi_{i j}(x)\right|_{\partial \Omega}
\end{gathered}
$$

Since $\delta \phi_{i j}(x)$ has compact support, $\delta_{i j}(x)=0$ on $\partial \Omega$. This immediately leads to Eq. (2). The vanishing boundary conditions are then established as

$$
\left.\frac{\partial^{j-i-1} w}{\partial x^{j-i-1}} \cdot \frac{\partial^{i}}{\partial x^{i}}\left(\frac{\partial v}{\partial\left(\frac{\partial w_{w}}{\partial x^{j}}\right)}\right)\right|_{\partial \Omega}=0
$$


for all $0 \leq i \leq j-1$ and $1 \leq j \leq m$.

It follows that

$$
f=-\sum_{i=0}^{m}(-1)^{i} \frac{\partial^{i}}{\partial x^{i}}\left(\frac{\partial v}{\partial\left(\frac{\partial^{i} w}{\partial x^{i}}\right)}\right)
$$

Consider the following general form of the potential energy density

$$
v=\sum_{i=0}^{m} \frac{1}{2} \frac{\partial^{i} w}{\partial x^{i}} \cdot k_{i}(x) \frac{\partial^{i} w}{\partial x^{i}}=\frac{1}{2} w \cdot k_{0}(x) w+\frac{1}{2} \frac{\partial w}{\partial x} \cdot k_{1}(x) \frac{\partial w}{\partial x}+\cdots+\frac{1}{2} \frac{\partial^{m} w}{\partial x^{m}} \cdot k_{m}(x) \frac{\partial^{m} w}{\partial x^{m}}
$$

where $k_{i}(x) \geq 0 \in \mathbb{R} \times \mathbb{R}, i=0,1, \ldots, m$. Note that $k_{i}(x)$ can be positive semi-definite but $v\left(x, w, \frac{\partial w}{\partial x}, \ldots \frac{\partial^{m} w}{\partial x^{m}}\right)$ is a positive-definite function.

We introduce the kinetic energy density as

$$
\tau=\frac{1}{2} \frac{\partial w}{\partial t} \cdot m(x) \frac{\partial w}{\partial t}
$$

Then,

$$
\delta w \cdot \frac{\partial}{\partial t}\left(\frac{\partial \tau}{\partial\left(\frac{\partial w}{\partial t}\right)}\right)=\delta w \cdot m(x) \frac{\partial^{2} w}{\partial t^{2}}
$$

The virtual work done on the system is expressed as

$$
\delta w \cdot\left[\frac{\partial}{\partial t}\left(\frac{\partial \tau}{\partial\left(\frac{\partial w}{\partial t}\right)}\right)+\sum_{i=0}^{m}(-1)^{i} \frac{\partial^{i}}{\partial x^{i}}\left(\frac{\partial v}{\partial\left(\frac{\partial{ }^{i} w}{\partial x^{i}}\right)}\right)-q\right]=0
$$

The Lagrangian density is formulated as

$$
\mathscr{L}=\tau-v=\frac{1}{2} \frac{\partial w}{\partial t} \cdot m(x) \frac{\partial w}{\partial t}-\sum_{i=0}^{m} \frac{1}{2} \frac{\partial^{i} w}{\partial x^{i}} \cdot k_{i}(x) \frac{\partial^{i} w}{\partial x^{i}}
$$

This leads to the Euler-Lagrange equation for the Lagrangian system (S) as

$$
\frac{\partial}{\partial t}\left(\frac{\partial \mathscr{L}}{\partial\left(\frac{\partial w}{\partial t}\right)}\right)-\sum_{i=0}^{m}(-1)^{i} \frac{\partial^{i}}{\partial x^{i}}\left(\frac{\partial \mathscr{L}}{\partial\left(\frac{\partial^{i} w}{\partial x^{i}}\right)}\right)-q=0
$$

Let $\mathscr{H}$ be a Hilbert space with the an inner product definition

$$
\langle u, v\rangle=\int_{\Omega} v \cdot m(x) u d x
$$

on a Lebesgue square integrable inner product space $\mathscr{L}^{2}(\Omega)$ where $u(x) \in \mathscr{H}(\Omega)$ and $v(x) \in \mathscr{H}(\Omega)$. Let $L: \mathscr{H} \rightarrow \mathscr{H}$ be a linear operator on $\mathscr{H}(\Omega)$ defined by

$$
L=m^{-1}(x) \sum_{i=0}^{m}(-1)^{i} \frac{\partial^{i}}{\partial x^{i}}\left(k_{i}(x) \frac{\partial^{i}}{\partial x^{i}}\right)
$$

Theorem 1: Let $L^{*}: \mathscr{H} \rightarrow \mathscr{H}$ be an linear operator on $\mathscr{H}(\Omega)$. Then, $L$ is a formally self-adjoint operator such that

$$
\langle v, L u\rangle=\left\langle u, L^{*} v\right\rangle
$$

Moreover, $L$ is a self-adjoint operator if the boundary conditions for $L$ and $L^{*}$ are equivalent. ${ }^{11}$ 
Proof: $\langle v(x), L u(x)\rangle$ is expressed as

$$
\begin{aligned}
&\langle v, L u\rangle=\int_{\Omega} v \cdot \sum_{i=0}^{m}(-1)^{i} \frac{\partial^{i}}{\partial x^{i}}\left(k_{i}(x) \frac{\partial^{i} u}{\partial x^{i}}\right) d x \\
&=\int_{\Omega} v \cdot\left[k_{0}(x) u-\frac{\partial}{\partial x}\left(k_{1}(x) \frac{\partial u}{\partial x}\right)+\frac{\partial^{2}}{\partial x^{2}}\left(k_{2}(x) \frac{\partial^{2} u}{\partial x^{2}}\right)+\cdots+(-1)^{m} \frac{\partial^{m}}{\partial x^{m}}\left(k_{m}(x) \frac{\partial^{m} u}{\partial x^{m}}\right)\right] d x
\end{aligned}
$$

Upon successive integration by parts, we get

$$
\begin{gathered}
\langle v, L u\rangle=\int_{\Omega} u \cdot\left[k_{0}(x) v-\frac{\partial}{\partial x}\left(k_{1}(x) \frac{\partial v}{\partial x}\right)+\frac{\partial^{2}}{\partial x^{2}}\left(k_{2}(x) \frac{\partial^{2} v}{\partial x^{2}}\right)+\cdots+(-1)^{m} \frac{\partial^{m}}{\partial x^{m}}\left(k_{m}(x) \frac{\partial^{m} v}{\partial x^{m}}\right)\right] d x \\
-\left[v \cdot k_{1}(x) \frac{\partial u}{\partial x}-u \cdot k_{1}(x) \frac{\partial v}{\partial x}-v \cdot \frac{\partial}{\partial x}\left(k_{2}(x) \frac{\partial^{2} u}{\partial x^{2}}\right)+\frac{\partial v}{\partial x} \cdot k_{2}(x) \frac{\partial^{2} u}{\partial x^{2}}-\frac{\partial u}{\partial x} \cdot k_{2}(x) \frac{\partial^{2} v}{\partial x^{2}}+u \cdot \frac{\partial}{\partial x}\left(k_{2}(x) \frac{\partial^{2} v}{\partial x^{2}}\right)\right. \\
\left.+\cdots+(-1)^{m-1} v \cdot \frac{\partial^{m-1}}{\partial x^{m-1}}\left(k_{m}(x) \frac{\partial^{m} u}{\partial x^{m}}\right)-\cdots-(-1)^{m-1} u \cdot \frac{\partial^{m-1}}{\partial x^{m-1}}\left(k_{m}(x) \frac{\partial^{m} v}{\partial x^{m}}\right)\right]_{\partial \Omega} \\
=\int_{\Omega} u \cdot \sum_{i=0}^{m}(-1)^{i} \frac{\partial^{i}}{\partial x^{i}}\left(k_{i}(x) \frac{\partial^{i} v}{\partial x^{i}}\right) d x-\sum_{j=1}^{m} \sum_{i=0}^{j-1}(-1)^{i}\left[\frac{\partial^{j-i-1} v}{\partial x^{j-i-1}} \cdot \frac{\partial^{i}}{\partial x^{i}}\left(k_{j}(x) \frac{\partial^{j} u}{\partial x^{j}}\right)-\frac{\partial^{j-i-1} u}{\partial x^{j-i-1}} \cdot \frac{\partial^{i}}{\partial x^{i}}\left(k_{j}(x) \frac{\partial^{j} v}{\partial x^{j}}\right)\right]_{\partial \Omega}
\end{gathered}
$$

The vanishing boundary term in Eq. (18) results in the following boundary conditions associated with the adjoint operator $L^{*}$ :

$$
\left.\frac{\partial^{j-i-1} v}{\partial x^{j-i-1}} \cdot \frac{\partial^{i}}{\partial x^{i}}\left(k_{j}(x) \frac{\partial^{j} v}{\partial x^{j}}\right)\right|_{\partial \Omega}=0
$$

Then,

$$
\langle v, L u\rangle=\left\langle u, L^{*} v\right\rangle=\int \sum_{\Omega} \sum_{i=0}^{m} u \cdot(-1)^{i} \frac{\partial^{i}}{\partial x^{i}}\left(k_{i}(x) \frac{\partial^{i} v}{\partial x^{i}}\right) d x
$$

Therefore,

$$
L^{*}=L=m^{-1}(x) \sum_{i=0}^{m}(-1)^{i} \frac{\partial^{i}}{\partial x^{i}}\left(k_{i}(x) \frac{\partial^{i}}{\partial x^{i}}\right)
$$

Thus, $L$ is a self-adjoint operator.

We note that $L$ is a positive-definite operator since

$$
\langle w, L w\rangle=2 \int_{\Omega} v d x>0
$$

The eigenvalue problem is given by

$$
L \phi=\lambda \phi
$$

It follows that

$$
\langle\phi, L \phi\rangle=\lambda\langle\phi, \phi\rangle
$$

Therefore, the eigenvalues $\lambda$ of $L$ are positive-definite since

$$
\lambda \doteq \omega^{2}=\frac{\langle\phi, L \phi\rangle}{\langle\phi, \phi\rangle}>0
$$

and its eigenfunctions $\left\{\phi_{i}(x)\right\}_{i=1}^{\infty}$ form a set of orthogonal basis functions such that $\left\langle\phi_{i}(x), \phi_{j}(x)\right\rangle=m_{i j} \delta_{i j}$ where $m_{i j}$ is a constant and $\delta_{i j}$ is the Kronecker delta.

Thus, the Lagrangrian system (S) can be expressed in an linear operator form as

$$
m(x) \frac{\partial^{2} w}{\partial t^{2}}+m(x) L w=q
$$




\section{Hamilton's Principle for Control of Lagrangian Systems}

Let $p(x, t): \mathbb{R} \times[0, \infty) \rightarrow \mathbb{R}$ be defined as a momentum density ${ }^{8}$ where

$$
p=\frac{\partial \mathscr{L}}{\partial\left(\frac{\partial w}{\partial t}\right)}=m(x) \frac{\partial w}{\partial t}
$$

The Hamiltonian density $h(x, t): \mathbb{R} \times[0, \infty) \rightarrow \mathbb{R}$ of the Lagrangian system (S) is defined as ${ }^{8}$

$$
h=\frac{\partial w}{\partial t} \cdot p-\mathscr{L}
$$

Substituting in Eq. (27) yields

$$
h=\frac{\partial w}{\partial t} \cdot m(x) \frac{\partial w}{\partial t}-\tau+v=\tau+v
$$

The Hamiltonian function is the integral of the Hamiltonian function

$$
H=\int_{\Omega}(\tau+v) d x
$$

The Lagrangian system (S) can be described the Hamilton's equations in the form

$$
\begin{gathered}
\frac{\partial w}{\partial t}=\frac{\partial h}{\partial p} \\
\frac{\partial p}{\partial t}=-m(x) L w+q
\end{gathered}
$$

The time derivative of the Hamiltonian function, or the integral of the Hamiltonian density, is evaluated as

$$
\dot{H}=\int_{\Omega} \dot{h} d x=\int_{\Omega}(\dot{\tau}+\dot{v}) d x=\int_{\Omega} \frac{\partial w}{\partial t} \cdot \frac{\partial}{\partial t}\left(\frac{\partial \mathscr{L}}{\partial\left(\frac{\partial w}{\partial t}\right)}\right) d x-\int_{\Omega} \frac{\partial}{\partial t}\left(\frac{\partial^{i} w}{\partial x^{i}}\right) \cdot \sum_{i=0}^{m} \frac{\partial^{i}}{\partial x^{i}} \frac{\partial \mathscr{L}}{\partial\left(\frac{\partial^{i} w}{\partial x^{i}}\right)} d x
$$

The second integral can be evaluated by integration by parts as

$$
\int_{\Omega} \frac{\partial}{\partial t}\left(\frac{\partial^{i} w}{\partial x^{i}}\right) \cdot \sum_{i=0}^{m} \frac{\partial^{i}}{\partial x^{i}} \frac{\partial \mathscr{L}}{\partial\left(\frac{\partial^{i} w}{\partial x^{i}}\right)} d x=\int_{\Omega} \frac{\partial w}{\partial t} \cdot \sum_{i=0}^{m}(-1)^{i} \frac{\partial^{i}}{\partial x^{i}}\left(\frac{\partial \mathscr{L}}{\partial\left(\frac{\partial^{i} w}{\partial x^{i}}\right)}\right) d x
$$

with the vanishing boundary terms due to the compact support of $\delta \phi_{i j}(x)$, that is,

$$
\left.\frac{\partial^{j-i-1} w}{\partial x^{j-i-1}} \cdot \frac{\partial}{\partial t} \frac{\partial^{i}}{\partial x^{i}}\left(\frac{\partial v}{\partial\left(\frac{\partial^{j} w}{\partial x^{j}}\right)}\right)\right|_{\partial \Omega}=0
$$

Thus, the time derivative of the Hamiltonian function becomes

$$
\dot{H}=\int_{\Omega} \frac{\partial w}{\partial t} \cdot\left[m(x) \frac{\partial^{2} w}{\partial t^{2}}+m(x) L w\right] d x=\int_{\Omega} \frac{\partial w}{\partial t} \cdot q d x
$$

If the non-conservative distributed force is zero, then the time derivative of the Hamiltonian function is zero which is the equivalence of the conservation of the total mechanical energy of a mechanical system. Thus, stability of the system can be inferred by the Hamilton's principle which is equivalent to the energy principle of a mechanical system. If energy is added to the system to increase the time rate of change of the work done by the non-conservative distributed force, or equivalently $\dot{H}(t)>0$, then the motion of the system would grow. Conversely, if the time rate of change of the work done by the non-conservative distributed force is negative, or equivalently $\dot{H}(t) \leq 0$, the system would exhibit a decayed or bounded motion which implies stability. The system is then said to be dissipative. 
A distributed control can be designed to ensure that the time derivative of the Hamiltonian function is negative. Many types of control are possible. Some common types of control include distributed control, discrete control, and boundary control. We will not consider the boundary control in the present study.

The non-conservative distributed force $q\left(x, t, w, \frac{\partial w}{\partial x}, \ldots, \frac{\partial^{m-1} w}{\partial x^{m-1}}, \frac{\partial w}{\partial t}, u\right)$ can be expressed by the individual contributions as

$$
q=q_{c}+b u
$$

where $q_{c}\left(x, t, w, \frac{\partial w}{\partial x}, \ldots, \frac{\partial^{m-1} w}{\partial x^{m-1}}, \frac{\partial w}{\partial t}\right)$ is a non-conservative distributed force without the distributed control $u(x, t)$ : $\mathbb{R} \times[0, \infty) \rightarrow \mathbb{R}$. The control sensitivity function $b(x) \in \mathbb{R}^{n}$ is spatially distributed. Then, the Hamilton's equation for the Lagrangian system $(\mathrm{S})$ is expressed as

$$
\frac{\partial p}{\partial t}=-m(x) L w+q_{c}+b u
$$

Practical applications of distributed control are very limited in general. For systems with truly distributed control, control actuation systems must be able to possess infinitesimal local control authorities at every single spatial location throughout a system. Such requirements are impossible to be met in practical applications. Due to the physical limitation and sizes of control actuation systems, only finite actuation systems can be physically implemented even if the sensing is distributed. We refer to this class of distributed control systems as discrete control systems. The Hamilton's equation for the Lagrangian system (S) with discrete control can be established as

$$
\frac{\partial p}{\partial t}=-m(x) L w+q_{c}+\sum_{i=1}^{p} b_{i} u_{i}
$$

where $b_{i}(x) \in \mathbb{R}^{n \times p}$ is a control sensitivity function with $p \geq n$ and $u_{i}(t)$ is a discrete control defined over an interval $a_{c_{i}} \leq x \leq b_{c_{i}}, i=1, \ldots, p$. A discrete control system thus only have a finite countable number of physical control actuation systems over the domain of a distributed system. Each of these control actuation systems acts on a subspace of the domain of the system and is commanded by a temporal command. A example of a discrete control system acting on a distributed system is a flap system on an aircraft wing.

Consider these two cases:

\section{A. Distributed Control}

A distributed control is a control that is spatially distributed and is denoted as $u(x, t)$. Then, $\dot{H}(t)$ is expressed as

$$
\dot{H}=\int_{\Omega} \frac{\partial w}{\partial t} \cdot q_{c} d x+\int_{\Omega_{c}} \frac{\partial w}{\partial t} \cdot b(x) u(x, t) d x
$$

where $\Omega_{c}=\left(a_{c}, b_{c}\right) \subseteq \Omega$ is a subspace on which the distributed control acts.

From the spectral theory, ${ }^{11}$ the general solution of $w(x, t)$ can be expressed in the form

$$
w(x, t)=\sum_{i=1}^{\infty} \phi_{i}(x) \theta_{i}(t)=\phi(x) \theta(t)
$$

where $\phi_{i}(x): \Omega \rightarrow \mathbb{R}^{n}$ is an eigenfunction of $(L-\lambda) \phi=0$ in the inner product space $\mathscr{L}^{2}(\Omega)$ and $\theta_{i}(t):[0, \infty) \rightarrow \mathbb{R}$. The infinite matrices $\phi(x): \Omega \rightarrow \mathbb{R}^{n \times \infty}$ and $\theta(t):[0, \infty) \rightarrow \mathbb{R}^{\infty}$ are an abstract representation of the infinite series solution of $w(x, t)$. The infinite matrix representation is convergent for the obvious reason that the infinite series converges to the solution of $w(x, t)$.

The distributed control is proposed to be formed by an infinite series solution

$$
u(x, t)=\sum_{j=1}^{\infty} \alpha_{j}(x) \beta_{j}(t)=\alpha(x) \beta(t)
$$

where $\alpha(x)=\left\{\alpha_{j}(x)\right\}_{j=1}^{\infty} \in \mathbb{R}^{\infty}$ and $\beta(t)=\left\{\beta_{j}(t)\right\}_{j=1}^{\infty} \in \mathbb{R}^{\infty}$ are infinite row and column vectors, respectively. For example, $\alpha(x)=\left\{\sin k_{j} x\right\}_{j=1}^{\infty}$ represents a Fourier sine series solution. 
$\dot{H}(t)$ is expressed in an infinite matrix form as

$$
\dot{H}=\sum_{i=1}^{\infty} \dot{\theta}_{i}\left[\int_{\Omega} \phi_{i} \cdot q_{c} d x+\int_{\Omega_{c}} \phi_{i} \cdot b(x) u(x, t) d x\right]=\theta^{\top}\left(\int_{\Omega} \phi^{\top} q_{c} d x+\int_{\Omega_{c}} \phi^{\top} b \alpha d x \beta\right)
$$

Let $B=\left\{\int_{\Omega_{c}} \phi_{i} \cdot b \alpha_{j} d x\right\}_{i, j=1}^{\infty} \in \mathbb{R}^{\infty \times \infty}$ an infinite square matrix. Then, the distributed control can be designed abstractly with the following choice of $\beta(t)$ :

$$
\beta=-B^{-1} \int_{\Omega} \phi^{\top}\left[q_{c}+m(x) c \frac{\partial w}{\partial t}-\mu m(x) L w\right] d x
$$

where $c>0 \in \mathbb{R}^{n \times n}$ is a velocity feedback gain matrix and $\mu \in \mathbb{R}$ is a displacement feedback gain. The existence of an infinite matrix inverse generally is not guaranteed. The notion of the determinant of an infinite matrix does not exist. The choice of the vector function $\alpha(x)$ influences $\beta(t)$. If $\mathscr{W} \in \mathscr{H}(\Omega)$ denotes the subspace of the solution $(L-\lambda) \phi_{i}(x)=0$, then $b(x) \alpha(x)$ must not exist in the orthogonal complement $\mathscr{W}^{\perp} \in \mathscr{H}(\Omega)$.

Consider the following example of an infinite matrix inverse in a Hilbert space. Let $f(x) \in \mathscr{H}(\Omega)$ be a function in $\mathscr{H}(\Omega)$ that admits an inner product and a linear operator $L$ with a complete set of orthonormal basis functions $\left\{\phi_{i}(x)\right\}_{i=1}^{\infty}$ that span $\mathbb{R}$. Then, we write $f(x)=\sum_{i=1}^{\infty} a_{i} \phi_{i}(x)$ where $a_{i} \doteq\left\langle f(x), \phi_{i}(x)\right\rangle=\int_{\Omega} f(x) \phi_{i}(x) d x$. Then, the norm square of $f(x)$ is expressed as

$$
\langle f, f\rangle=\|f\|^{2}=\int_{\Omega} f^{2} d x=\int_{\Omega} \sum_{i=1}^{\infty} \sum_{j=1}^{\infty} a_{i} \phi_{i} \cdot a_{j} \phi_{j} d x=a \int_{\Omega} \phi \phi^{\top} d x a^{\top}
$$

where $a=\left\{a_{i}\right\}_{i=1}^{\infty}$ and $\phi=\left\{\phi_{i}(x)\right\}_{i=1}^{\infty}$. But $\|f\|^{2}=\sum_{i}^{\infty} a_{i}^{2}=a a^{\top}$. Let $A=\left\{\int_{\Omega} \phi_{i}(x) \phi_{j}(x) d x\right\}_{i, j=1}^{\infty}=\int_{\Omega} \phi \phi^{\top} d x \in$ $\mathbb{R}^{\infty \times \infty}$. Then, we write $\|f\|^{2}=a A A^{-1} a^{\top}$. By comparing to Eq. (45), we see that $A^{-1}=I_{\infty \times \infty}$, which is an infinite identity matrix. We can also use the orthonormal property to show the same by noting that $A=\left\{\delta_{i j}\right\}_{i, j=1}^{\infty}=I_{\infty \times \infty}$ since $\int_{\Omega} \phi_{i}(x) \phi_{j}(x) d x=\delta_{i j}$.

The abstract form of the distributed control in Eq. (44) does not have practical applications. Instead, consider a weak formulation of the Lagrangian system (S) with $v(x, t) \in \mathscr{W}^{m}=H^{m}(\Omega)$ where $H^{m}(\Omega)$ is a Sobolev space with a weak derivative notion ${ }^{2}$

$$
\int_{\Omega} v \frac{\partial^{m} \varphi}{\partial x^{m}} d x=-\int_{\Omega} \frac{\partial^{m} v}{\partial x^{m}} \varphi d x
$$

where $\varphi(x) \in C_{c}^{\infty}(\Omega)$ is a smooth infinitely differentiable test function with compact support in $\Omega$. Then, $v(x, t)$ is a weak-form solution that approximates the strong-form solution of $w(x, t)$. We write

$$
v(x, t)=\sum_{i=1}^{N} \varphi_{i}(x) \vartheta_{i}(t)=\varphi(x) \vartheta(t)
$$

where $\varphi_{i}(x): \Omega \rightarrow \mathbb{R}^{n}$ is a trial orthogonal basis function in the inner product space $\mathscr{L}^{2}(\Omega)$ and $\vartheta_{i}(t):[0, \infty) \rightarrow \mathbb{R}$, satisfying the weak form of Eq. (26)

$$
\left\langle\varphi_{i}, \frac{\partial^{2} v}{\partial t^{2}}\right\rangle+\left\langle\varphi_{i}, L v\right\rangle=\left\langle\varphi_{i}, m^{-1} q\right\rangle
$$

for $i=1, \ldots, N$. Then, $v(x, t) \rightarrow w(x, t)$ as $N \rightarrow \infty$. Thus, $v(x, t)=w(x, t)+\varepsilon(N)$ where $\varepsilon(N)$ is an approximation error such that $\varepsilon(N) \rightarrow 0$ as $N \rightarrow \infty$.

The weak-form expression can be expressed as

$$
\left[\int_{\Omega} \varphi^{\top} m(x) \varphi d x\right] \ddot{\vartheta}+\left[\sum_{i=0}^{m} \int_{\Omega} \frac{\partial^{i} \varphi^{\top}}{\partial x^{i}} k_{i}(x) \frac{\partial^{i} \varphi}{\partial x^{i}} d x\right] \vartheta=\int_{\Omega} \varphi^{\top} q d x
$$

Let $u(x, t)$ be approximated by a truncated finite series solution

$$
u(x, t)=\sum_{j=1}^{p} \alpha_{j}(x) \beta_{j}(t)=\alpha(x) \beta(t)
$$


where $p \geq N$. The infinite matrix $B$ is now replaced by a finite matrix $B=\left\{\int_{\Omega_{c}} \varphi_{i} \cdot b \alpha_{j} d x\right\} \in \mathbb{R}^{N \times p}$. Then, the weak-form distributed control can be designed concretely with the following choice of $\beta(t)$ :

$$
\beta=-B^{\top}\left(B B^{\top}\right)^{-1} \int_{\Omega} \varphi^{\top}\left[q_{c}+m(x) c \frac{\partial w}{\partial t}-\mu m(x) L w\right] d x
$$

assuming the choice of $\alpha(x)$ results in the existence of the matrix inverse $\left(B B^{\top}\right)^{-1}$.

Before we close this discussion, consider an alternative strong-form distributed control design. Let $B: \Omega_{c} \rightarrow \mathbb{R}^{p}$ such that

$$
B=\int_{\Omega_{c}} \frac{\partial w}{\partial t} \cdot b \alpha d x
$$

Then, the distributed control can be designed with

$$
\beta=-B^{\top}\left(B B^{\top}\right)^{-1} \int_{\Omega} \frac{\partial w}{\partial t} \cdot\left[q_{c}+m(x) c \frac{\partial w}{\partial t}-\mu m(x) L w\right] d x
$$

This strong-form distributed control is a nonlinear control, which can cause difficulty, whereas the weak-form distributed control in Eq. (51) is a linear control, which is much easier to handle with.

We now assert the stability of the Lagrangian system $(\mathrm{S})$ with the distributed control by the following theorem:

Theorem 2: The weak-form distributed control of Eq. (51) is exponentially stable and results in $w(x, t) \rightarrow 0$ as $N \rightarrow \infty$ for $\mu<1-\left(\frac{c}{2 \omega_{i}}\right)^{2}<1$ where $c$ is a constant.

Proof: Substituting Eq. (51) into Eq. (40) yields

$$
\begin{aligned}
& \dot{H}=\int_{\Omega} \frac{\partial w}{\partial t} \cdot q_{c} d x-\int_{\Omega} \frac{\partial v}{\partial t} \cdot {\left[q_{c}+m(x) c \frac{\partial w}{\partial t}-\mu m(x) L w\right] d x } \\
&=-\int_{\Omega} \frac{\partial \varepsilon}{\partial t} \cdot q_{c} d x-\int_{\Omega}\left(\frac{\partial w}{\partial t}+\frac{\partial \varepsilon}{\partial t}\right) \cdot\left[m(x) c \frac{\partial w}{\partial t}-\mu m(x) L w\right] d x \\
& \leq-\int_{\Omega} \frac{\partial w}{\partial t} \cdot m(x) c \frac{\partial w}{\partial t} d x+\mu \dot{H}+\left\|\frac{\partial \varepsilon}{\partial t}\right\|\left\|q_{c}+m(x) c \frac{\partial w}{\partial t}-\mu m(x) L w\right\|
\end{aligned}
$$

$\dot{H}(t)$ is then bounded by

$$
\dot{H} \leq-\int_{\Omega} \frac{\partial w}{\partial t} \cdot m(x) c \frac{\partial w}{\partial t} d x+\mu \dot{H}+c_{1}\left\|\frac{\partial w}{\partial t}\right\|+c_{2}\|w\|+c_{3}
$$

where $c_{1}=\left\|\frac{\partial \varepsilon}{\partial t}\right\|\|m(x) c\|, c_{2}=\left\|\frac{\partial \varepsilon}{\partial t}\right\| \frac{\|\mu m(x)\|\|L w\|}{\|w\|}$, and $c_{3}=\left\|\frac{\partial \varepsilon}{\partial t}\right\|\left\|q_{c}\right\|$. As $N \rightarrow \infty, v(x, t) \rightarrow w(x, t)$ and $\varepsilon(N) \rightarrow 0$. Thus, $\left\{c_{i}\right\}_{i=1}^{3} \rightarrow 0$ as $N \rightarrow \infty$. Then, $\dot{H}(t)$ becomes

$$
\dot{H} \leq-\int_{\Omega} \frac{\partial w}{\partial t} \cdot m(x) c \frac{\partial w}{\partial t} d x+\mu \dot{H}
$$

$\mu$ admits both positive and negative values. If $\mu \in[0,1)$, then

$$
(1-\mu) \dot{H} \leq-\int_{\Omega} \frac{\partial w}{\partial t} \cdot m(x) c \frac{\partial w}{\partial t} d x
$$

which implies

$$
\dot{H} \leq-\frac{1}{1-\mu} \int_{\Omega} \frac{\partial w}{\partial t} \cdot m(x) c \frac{\partial w}{\partial t} d x \leq-\int_{\Omega} \frac{\partial w}{\partial t} \cdot m(x) c \frac{\partial w}{\partial t} d x
$$

If $\mu<0$, then

$$
\dot{H} \leq(1-\mu) \dot{H} \leq-\int_{\Omega} \frac{\partial w}{\partial t} \cdot m(x) c \frac{\partial w}{\partial t} d x
$$

Therefore, $\dot{H}(t)$ is bounded by

$$
\dot{H} \leq-\int_{\Omega} \frac{\partial w}{\partial t} \cdot m(x) c \frac{\partial w}{\partial t} d x
$$


Thus, $\dot{H}(t) \leq 0$ for $\mu<1$. It follows that $H(t) \in \mathscr{L}_{\infty}$ is bounded and so is $w(x, t) \in \mathscr{L}_{\infty}$. It also implies that the $\mathscr{L}_{2}$ norm of $\frac{\partial w(x, t)}{\partial t}$ exists. Therefore, $\frac{\partial w(x, t)}{\partial t} \in \mathscr{L}_{2} \cap \mathscr{L}_{\infty}$. The time derivative of $\dot{H}(t)$ is evaluated as

$$
\ddot{H} \leq-2 \int_{\Omega} \frac{\partial w}{\partial t} \cdot m(x) c \frac{\partial^{2} w}{\partial t^{2}} d x
$$

Since $w(x, t) \in \mathscr{L}_{2} \cap \mathscr{L}_{\infty}, \ddot{H}(t) \in \mathscr{L}_{\infty}$ is bounded. It then follows from the Barbalat's lemma that $\dot{H}(t)$ is uniformly continuous and $\dot{H}(t) \rightarrow 0$ as $t \rightarrow \infty$. This implies $w(x, t) \rightarrow 0$ as $t \rightarrow \infty$. Thus, the distributed control is asymptotically stable.

The general closed-loop solution of $w(x, t)$ can be expressed in the form

$$
w(x, t)=\sum_{i=1}^{\infty} \phi_{i}(x) e^{\lambda_{i} t}
$$

where $\lambda_{i} \in \mathbb{C}$. Then, $\dot{H}(t)$ is expressed as

$$
\dot{H}=-\sum_{i=1}^{\infty} \int_{\Omega} \lambda_{i} e^{\lambda_{i} t} \phi_{i} \cdot\left[m(x) c \phi_{i} \lambda_{i}-\mu m(x) L \phi_{i}\right] e^{\lambda_{i} t} d x
$$

But

$$
\dot{H}=\int_{\Omega} \frac{\partial w}{\partial t} \cdot\left[m(x) \frac{\partial^{2} w}{\partial t^{2}}+m(x) L w\right] d x=\sum_{i=1}^{\infty} \int_{\Omega} \lambda_{i} e^{\lambda_{i} t} \phi_{i} \cdot\left[m(x) \phi_{i} \lambda_{i}^{2}+m(x) L \phi_{i}\right] e^{\lambda_{i} t} d x
$$

Thus, expressing in terms of the inner product definition, we obtain

$$
\sum_{i=1}^{\infty}\left[\left\langle\phi_{i}, \phi_{i}\right\rangle \lambda_{i}^{2}+\left\langle\phi_{i}, c \phi_{i}\right\rangle \lambda_{i}+(1-\mu)\left\langle\phi_{i}, L \phi_{i}\right\rangle\right] \lambda_{i} e^{2 \lambda_{t} t}=0
$$

Without loss of generality, we assume $c$ is a constant and take advantage of the positive real eigenvalues of the symmetric self-adjoint operator $L$. We then obtain

$$
\lambda_{i}^{2}+c \lambda_{i}+(1-\mu) \omega_{i}^{2}=0
$$

which yields

$$
\lambda_{i}=-\frac{c}{2} \pm i \omega_{i} \sqrt{1-\mu-\left(\frac{c}{2 \omega_{i}}\right)^{2}}
$$

This implies exponential stability if $\mu<1-\left(\frac{c}{2 \omega_{i}}\right)^{2}$. Thus, $\dot{H}(t)$ is bounded by

$$
\dot{H} \leq-\int_{\Omega} \frac{\partial w}{\partial t} \cdot m(x) c \frac{\partial w}{\partial t} d x \leq-c \sum_{i=1}^{\infty}\left\langle\phi_{i}, \phi_{i}\right\rangle\left\|\lambda_{i}\right\|^{2}\left\|e^{ \pm i 2 \omega_{i} \sqrt{1-\mu-\left(\frac{c}{2 \omega_{i}}\right)^{2}} t}\right\| e^{-c t}
$$

Since $\left\|e^{ \pm i 2 \omega_{i} \sqrt{1-\mu-\left(\frac{c}{2 \omega_{i}}\right)^{2}} t}\right\| \leq 1$, we obtain

$$
\dot{H} \leq-c \sum_{i=1}^{\infty}\left\langle\phi_{i}, \phi_{i}\right\rangle\left\|\lambda_{i}\right\|^{2} e^{-c t}
$$

Therefore, the closed-loop Lagrangian system (S) is exponentially stable.

The strong-form distributed control of Eq. (53) requires a special consideration. To prevent the possibility of a control blow-up as $\frac{\partial w(x, t)}{\partial t} \rightarrow 0$ for which $\left(B B^{\top}\right)^{-1} \rightarrow \infty$, a modification to the strong-form distributed control 
is necessary. A dead-band method is applied whereby $\beta(0)$ whenever $\left\|\frac{\partial w}{\partial t}\right\| \leq \varepsilon$. Alternatively, the strong-form distributed control of Eq. (53) is replaced by the weak-form distributed control of Eq. (51) whenever $\left\|\frac{\partial w}{\partial t}\right\| \leq \varepsilon$.

Corollary 1: The strong-form distributed control of Eq. (53) results in bounded closed-loop signals if $\beta(t)=0$ or $\beta(t)$ is given by Eq. (51) for $\left\|\frac{\partial w}{\partial t}\right\| \leq \varepsilon$. Moreover, the strong-form distributed control is exponentially stable if $\beta(t)$ is given by Eq. (51) for $\left\|\frac{\partial w}{\partial t}\right\| \leq \varepsilon$ as $N \rightarrow \infty$.

Proof: Substituting Eq. (53) into Eq. (40) and using Ineq. (58), we get

$$
\dot{H} \leq-\int_{\Omega} \frac{\partial w}{\partial t} \cdot m(x) c \frac{\partial w}{\partial t} d x+\mu \dot{H} \leq-\int_{\Omega} \frac{\partial w}{\partial t} \cdot m(x) c \frac{\partial w}{\partial t} d x
$$

for $\left\|\frac{\partial w}{\partial t}\right\|>\varepsilon$ and

$$
\dot{H}=\int_{\Omega} \frac{\partial w}{\partial t} \cdot q_{c} d x \leq c_{4}
$$

for $\left\|\frac{\partial w}{\partial t}\right\| \leq \varepsilon$ if $\beta(t)=0$, where $c_{4}=\varepsilon\left\|q_{c}\right\|$.

If $\beta(t)$ is replaced by Eq. (51) for $\left\|\frac{\partial w}{\partial t}\right\| \leq \varepsilon$, then

$$
\dot{H} \leq-\int_{\Omega} \frac{\partial w}{\partial t} \cdot m(x) c \frac{\partial w}{\partial t} d x+\mu \dot{H}+c_{1} \varepsilon+c_{2}\|w\|+c_{3}
$$

Using Ineq. (58), Ineq. 72 becomes

$$
\dot{H} \leq-\int_{\Omega} \frac{\partial w}{\partial t} \cdot m(x) c \frac{\partial w}{\partial t} d x+\frac{1}{1-\mu}\left(c_{1} \varepsilon+c_{2}\|w\|+c_{3}\right)
$$

if $\mu \in[0,1)$ and

$$
\dot{H} \leq-\int_{\Omega} \frac{\partial w}{\partial t} \cdot m(x) c \frac{\partial w}{\partial t} d x+c_{1} \varepsilon+c_{2}\|w\|+c_{3}
$$

if $\mu<0$.

Thus, collectively $\dot{H}(t)$ is bounded by

$$
\dot{H} \leq-\int_{\Omega} \frac{\partial w}{\partial t} \cdot m(x) c \frac{\partial w}{\partial t} d x+\frac{1}{1-\mu}\left(c_{1} \varepsilon+c_{2}\|w\|+c_{3}\right) \leq c_{4}
$$

where $c_{4}=-\min (m(x) c) \varepsilon^{2}+\frac{1}{1-\mu}\left(c_{1} \varepsilon+c_{2}\|w\|+c_{3}\right)$.

Thus, $\dot{H}(t) \leq 0$ for $\left\|\frac{\partial w}{\partial t}\right\|>\varepsilon$ and $\dot{H}(t) \leq c_{4}$ for $\left\|\frac{\partial w}{\partial t}\right\| \leq \varepsilon$. Therefore, the closed-loop Lagrangian system (S) is bounded.

If $\beta(t)$ is replaced by Eq. (51), then from Theorem 2

$$
\dot{H} \leq-\int_{\Omega} \frac{\partial w}{\partial t} \cdot m(x) c \frac{\partial w}{\partial t} d x
$$

for $H(t) \leq \varepsilon$. Thus, collectively

$$
\dot{H} \leq-\int_{\Omega} \frac{\partial w}{\partial t} \cdot m(x) c \frac{\partial w}{\partial t} d x
$$

for all $H(t)$. Then, from Theorem 2, the closed-loop Lagrangian system (S) is exponentially stable.

\section{B. Discrete Control}

A discrete control is a control that is spatially independent and is denoted as $u_{i}(t), i=1, \ldots, p . \dot{H}(t)$ is expressed as

$$
\dot{H}=\int_{\Omega} \frac{\partial w}{\partial t} \cdot q_{c} d x+\sum_{i=1}^{p} \int_{\Omega_{c_{i}}} \frac{\partial w}{\partial t} \cdot b_{i}(x) u_{i} d x
$$


where $\Omega_{c_{i}}=\left(a_{c_{i}}, b_{c_{i}}\right) \subset \Omega, u(t)=\left[\begin{array}{lll}u_{1}(t) & \ldots & u_{p}(t)\end{array}\right]^{\top}$. The weak-form expression of $\dot{H}(t)$ is

$$
\dot{H}=\vartheta^{\top}\left[\int_{\Omega} \varphi^{\top} q_{c} d x+\sum_{i=1}^{p} \int_{\Omega_{c_{i}}} \varphi^{\top} b_{i}(x) u_{i} d x\right]
$$

as

Choose $\varphi(x) \in \mathbb{R}^{n \times N}$ with $N \leq p$. Let $B=\left\{\int_{\Omega_{c_{j}}} \varphi_{i} \cdot b_{j} d x\right\}_{i=1, j=1}^{i=N, j=p} \in \mathbb{R}^{N \times p}$. Then, the discrete control is obtained

$$
u=-B^{\top}\left(B B^{\top}\right)^{-1} \int_{\Omega} \varphi^{\top}\left[q_{c}+m(x) c \frac{\partial w}{\partial t}-\mu m(x) L w\right] d x
$$

The stability of the closed-loop distributed system can be proved by Corollary 1 .

To complete this section, we also present the strong-form discrete control

$$
u=-B^{\top}\left(B B^{\top}\right)^{-1} \int_{\Omega} \frac{\partial w}{\partial t} \cdot\left[q_{c}+m(x) c \frac{\partial w}{\partial t}-\mu m(x) L w\right] d x
$$

where $B=\left\{\int_{\Omega_{c_{i}}} \frac{\partial w}{\partial t} \cdot b_{i} d x\right\}_{i=1}^{p} \in \mathbb{R}^{p}$.

\section{Lyapunov's Method and Least-Action Distributed Control}

The Hamilton's principle is a special case of the Lyapunov's method where the Hamiltonian function represents the total energy of a system, and is a special and unique Lyapunov function. The Lyapunov's method states that, given a positive-definite Lyapunov candidate function $V(t)$, a system is stable if the Lie derivative $\dot{V}(t)$ evaluated on the system's trajectory is negative semi-definite. Any positive-definite function could be a candidate Lyapunov function, but a Lyapunov function must satisfy $\dot{V}(t) \leq 0$.

Given an arbitrary candidate Lyapunov function

$$
V=\int_{\Omega}(\tau+g) d x
$$

where $g\left(x, w, \frac{\partial w}{\partial x}, \ldots \frac{\partial^{m} w}{\partial x^{m}}\right)>0$, we can state the principle of least action for distributed control as follows.

Theorem 3: The distributed control $u(x, t)$ in Eq. (53) with $\mu=0$ that minimizes $\dot{V}(t)$ is a minimum-norm control if and only if $g\left(x, w, \frac{\partial w}{\partial x}, \ldots \frac{\partial^{m} w}{\partial x^{m}}\right)=v\left(x, w, \frac{\partial w}{\partial x}, \ldots \frac{\partial^{m} w}{\partial x^{m}}\right)$ which implies $V(t)=H(t)$.

Proof: Evaluating $\dot{V}(t)$ yields

$$
\begin{array}{r}
\dot{V}=\int_{\Omega}\left[\frac{\partial w}{\partial t} \cdot \frac{\partial}{\partial t}\left(\frac{\partial \tau}{\partial\left(\frac{\partial w}{\partial t}\right)}\right)+\sum_{i=0}^{m} \frac{\partial}{\partial t}\left(\frac{\partial^{i} w}{\partial x^{i}}\right) \cdot \frac{\partial^{i}}{\partial x^{i}}\left(\frac{\partial g}{\partial\left(\frac{\partial^{i} w}{\partial x^{i}}\right)}\right)\right] d x \\
=\int_{\Omega} \frac{\partial w}{\partial t} \cdot\left[q_{c}+\sum_{i=0}^{m}(-1)^{i} \frac{\partial^{i}}{\partial x^{i}}\left(\frac{\partial(g-v)}{\partial\left(\frac{\partial^{i} w}{\partial x^{i}}\right)}\right)\right] d x+\left.\sum_{j=1}^{m} \sum_{i=0}^{j-1}(-1)^{i} \frac{\partial}{\partial t}\left(\frac{\partial^{j-i-1} w}{\partial x^{j-i-1}}\right) \cdot \frac{\partial^{i}}{\partial x^{i}}\left(\frac{\partial g}{\partial\left(\frac{\partial^{j} w}{\partial x^{j}}\right)}\right)\right|_{\partial \Omega} \\
+\int_{0}^{L} \frac{\partial w}{\partial t} \cdot b(x) u(x, t) d x
\end{array}
$$

We formulate a distributed control law by letting $u(x, t)=\alpha(x) \beta(t)+\alpha(x) \Delta \beta(t)$. Then, $\dot{V}(t)$ can be expressed as

$$
\begin{array}{r}
\dot{V}=\dot{H}+\int_{\Omega} \sum_{i=0}^{m} \frac{\partial w}{\partial t} \cdot(-1)^{i} \frac{\partial^{i}}{\partial x^{i}}\left(\frac{\partial(g-v)}{\partial\left(\frac{\partial^{i} w}{\partial x^{i}}\right)}\right) d x+\left.\sum_{j=1}^{m} \sum_{i=0}^{j-1}(-1)^{i} \frac{\partial}{\partial t}\left(\frac{\partial^{j-i-1} w}{\partial x^{j-i-1}}\right) \cdot \frac{\partial^{i}}{\partial x^{i}}\left(\frac{\partial g}{\partial\left(\frac{\partial^{j} w}{\partial x^{j}}\right)}\right)\right|_{\partial \Omega} \\
+\int_{0}^{L} \frac{\partial w}{\partial t} \cdot b(x) \alpha(x) \Delta \beta d x
\end{array}
$$


The incremental control $\Delta \beta(t)$ is designed as

$\Delta \beta=-B^{\top}\left(B B^{\top}\right)^{-1}\left[\int_{\Omega} \frac{\partial w}{\partial t} \cdot \sum_{i=0}^{m}(-1)^{i} \frac{\partial^{i}}{\partial x^{i}}\left(\frac{\partial(g-v)}{\partial\left(\frac{\partial^{i} w}{\partial x^{i}}\right)}\right) d x+\left.\sum_{j=1}^{m} \sum_{i=0}^{j-1}(-1)^{i} \frac{\partial}{\partial t}\left(\frac{\partial^{j-i-1} w}{\partial x^{j-i-1}}\right) \cdot \frac{\partial^{i}}{\partial x^{i}}\left(\frac{\partial g}{\partial\left(\frac{\partial j_{w}}{\partial x^{j}}\right)}\right)\right|_{\partial \Omega}\right]$

Then,

$$
\dot{V}=\dot{H} \leq 0
$$

results in asymptotic stability.

It is obvious that, if $g=v, u(x, t)$ is a minimum since $\Delta \beta(t)=0$. We can pose the problem formally by seeking the function $g\left(x, w, \frac{\partial w}{\partial x}, \ldots \frac{\partial^{m} w}{\partial x^{m}}\right)$ that minimizes $\dot{V}(t)$. To that end, we apply calculus of variations by considering a variation $\delta g\left(x, w, \frac{\partial w}{\partial x}, \ldots \frac{\partial^{m} w}{\partial x^{m}}\right)$. Without loss of generality, we assume $u(x, t)=\alpha(x) \beta(t)$ remains fixed. Then, we compute the variation in $\dot{V}(t)$ due to the variation $\delta g\left(x, w, \frac{\partial w}{\partial x}, \ldots \frac{\partial^{m} w}{\partial x^{m}}\right)$ by expressing $\dot{V}(t)=\dot{H}(t)+\delta \dot{V}(t)$ where $\delta \dot{V}(t)$ is given by

$$
\delta \dot{V}=\int_{\Omega} \sum_{i=0}^{m}(-1)^{i} \frac{\partial w}{\partial t} \cdot \frac{\partial^{i}}{\partial x^{i}}\left(\frac{\partial(\delta g-\delta v)}{\partial\left(\frac{\partial^{i} w}{\partial x^{i}}\right)}\right) d x+\left.\sum_{j=1}^{m} \sum_{i=0}^{j-1}(-1)^{i} \frac{\partial}{\partial t}\left(\frac{\partial^{j-i-1} w}{\partial x^{j-i-1}}\right) \cdot \frac{\partial^{i}}{\partial x^{i}}\left(\frac{\partial \delta g}{\partial\left(\frac{\partial j_{w}}{\partial x^{j}}\right)}\right)\right|_{\partial \Omega}
$$

where

$$
\delta(\cdot)=\frac{\partial(\cdot)}{\partial w} \delta w+\frac{\partial(\cdot)}{\partial \frac{\partial w}{\partial x}} \delta \frac{\partial w}{\partial x}+\frac{\partial(\cdot)}{\partial \frac{\partial^{2} w}{\partial x^{2}}} \delta \frac{\partial^{2} w}{\partial x^{2}}=\sum_{i=0}^{m} \frac{\partial(\cdot)}{\partial \frac{\partial^{i} w}{\partial x^{i}}} \delta \frac{\partial^{i} w}{\partial x^{i}}
$$

The stationary condition for minimizing $\dot{V}(t)$ requires $\delta \dot{V}(t)=0$ for any arbitrary variation $\delta g\left(x, w, \frac{\partial w}{\partial x}, \ldots \frac{\partial^{m} w}{\partial x^{m}}\right)$. This immediately leads to $g\left(x, w, \frac{\partial w}{\partial x}, \ldots \frac{\partial^{m} w}{\partial x^{m}}\right)=v\left(x, w, \frac{\partial w}{\partial x}, \ldots \frac{\partial^{m} w}{\partial x^{m}}\right)$ and $V(t)=H(t)$

The advantage of the Lyapunov's method by not requiring the knowledge of the Hamiltonian function can sometimes be a weakness. It tends to lead to a more complicated control law design which also may result in more control effort. On the other hand, the Hamilton's principle requires the physical insight of a system but the control design task tends to be simpler and result in less control effort.

\section{Adaptive Control}

We consider an uncertain Lagrangian system (W) described by

$$
m(x) \frac{\partial^{2} w}{\partial t^{2}}+m(x) L w=q_{c}+\Delta q_{c}+b u
$$

where $\Delta q_{c}\left(x, w, \frac{\partial w}{\partial x}, \ldots, \frac{\partial^{m-1} w}{\partial x^{m-1}}, \frac{\partial w}{\partial t}\right) \in \mathbb{R}^{n}$ represents the system uncertainty, $b(x)=\left[\begin{array}{lll}b_{1}(x) & \cdots & b_{p}(x)\end{array}\right] \in \mathbb{R}^{n \times p}$ is a control sensitivity vector with $p \geq n, u(t)=\left[\begin{array}{lll}u_{1}(t) & \cdots & u_{p}(t)\end{array}\right]^{\top} \in \mathbb{R}^{p}$ is a vector of discrete controls.

The goal is to design an adaptive controller that can track a reference model described by

$$
m(x) \frac{\partial^{2} w_{m}}{\partial t^{2}}+m(x) L w_{m}=b u_{m}+b r
$$

where $r(t):[0, \infty) \rightarrow \mathbb{R}^{p} \in \mathscr{L}_{\infty}$ is a vector of bounded reference command signals and

$$
u_{m}=-B^{\top}\left(B B^{\top}\right)^{-1} \int_{\Omega} \varphi^{\top}\left[m(x) c \frac{\partial w_{m}}{\partial t}-\mu m(x) L w_{m}\right] d x
$$


We design an adaptive controller as

$$
u=u_{\text {nom }}+u_{a d}
$$

where $u_{\text {nom }}(t)$ is a nominal controller and $u_{a d}(t)$ is an adaptive augmentation controller to effectively cancel out the uncertainty. The nominal controller $u_{\text {nom }}(t)$ can be designed as

$$
u_{\text {nom }}=-B^{\top}\left(B B^{\top}\right)^{-1} \int_{\Omega} \varphi^{\top}\left[q_{c}+m(x) c \frac{\partial w}{\partial t}-\mu m(x) L w\right] d x+r
$$

Then, in the ideal case when $\Delta q_{c}=0$, the closed-loop distributed system tends to the reference model with $w(x, t) \rightarrow w_{m}(x, t)$ as $t \rightarrow \infty$.

The parametric uncertainty is expressed as

$$
\Delta q_{c}=\Phi^{\top}(x, t) \Theta(x)+d(x, t)
$$

where $\Phi\left(x, w, \frac{\partial w}{\partial x}, \ldots, \frac{\partial^{m-1} w}{\partial x^{m-1}}, \frac{\partial w}{\partial t}\right) \in \mathbb{R}^{q \times n}$ is a matrix of regressor functions, $\Theta(x) \in \mathbb{R}^{q}$ is a vector of unknown distributions, and $d(x, t)$ is an exogenous disturbance. We parameterize $\Theta(x)$ as

$$
\Theta=\gamma^{\top}(x) a
$$

where $\gamma(x) \in \mathbb{R}^{r \times q}$ is a matrix of prescribed distributions and $a \in \mathbb{R}^{r}$ is a vector of unknown constant parameters.

We approximate the disturbance as

$$
d=\sum_{i=1}^{N} \varphi_{i}(x) \sigma_{i}(t)=\varphi(x) \sigma(t)
$$

where $\sigma(t) \in \mathscr{L}_{\infty}$ is a bounded signal with a bounded time derivative such that $\|\dot{\sigma}(t)\| \leq \delta$.

Let $\hat{a}(t)$ and $\hat{\sigma}(t)$ be the estimates of $a$ and $\sigma(t)$, respectively. Then, the adaptive augmentation controller is designed as

$$
u_{a d}=-B^{\top}\left(B B^{\top}\right)^{-1} \int_{\Omega} \varphi^{\top}\left(\Phi^{\top} \gamma^{\top} \hat{a}+\varphi \hat{\sigma}\right) d x
$$

We define the tracking error as $e(x, t)=w(x . t)-w_{m}(x, t)$. The error equation is then formulated as

$$
\begin{aligned}
m(x) \frac{\partial^{2} e}{\partial t^{2}}+m(x) L e=q_{c}+\Phi^{\top} \gamma^{\top} a & +\varphi(x) \sigma(t) \\
& -b B^{\top}\left(B B^{\top}\right)^{-1} \int_{\Omega} \varphi^{\top}\left[q_{c}+m(x) c \frac{\partial e}{\partial t}-\mu m(x) L e+\Phi^{\top} \gamma^{\top} \hat{a}+\varphi \hat{\sigma}\right] d x
\end{aligned}
$$

The Hamiltonian of the error equation is expressed as

$$
H=\int_{\Omega}\left[\frac{1}{2} \frac{\partial e}{\partial t} \cdot m(x) \frac{\partial e}{\partial t}+\sum_{i=0}^{m} \frac{1}{2} \frac{\partial^{i} e}{\partial x^{i}} \cdot k_{i}(x) \frac{\partial^{i} e}{\partial x^{i}}\right] d x
$$

Let $e(x, t)=\varphi(x) \vartheta_{e}(t)-\varepsilon(N)$. Then,

$$
\dot{H}=\int_{\Omega} \frac{\partial e}{\partial t} \cdot\left[q_{c}+\Phi^{\top} \gamma^{\top} a+\varphi(x) \sigma(t)\right] d x-\dot{\vartheta}_{e}^{\top} \int_{\Omega} \varphi^{\top}\left[q_{c}+m(x) c \frac{\partial e}{\partial t}-\mu m(x) L e+\Phi^{\top} \gamma^{\top} \hat{a}+\varphi \hat{\sigma}\right] d x
$$

where $\tilde{a}_{i}(t)=\hat{a}_{i}(t)-a_{i}$ and $\tilde{\sigma}(t)=\hat{\sigma}(t)-\sigma(t)$ are the estimation errors of $\hat{a}(t)$ and $\hat{\sigma}(t)$, respectively.

Using the result of Theorem $2, \dot{H}(t)$ is bounded by

$$
\dot{H} \leq-\int_{\Omega} \frac{\partial e}{\partial t} \cdot\left[m(x) c \frac{\partial e}{\partial t}+\Phi^{\top} \gamma^{\top} \tilde{a}+\varphi \tilde{\sigma}\right] d x+c_{1}\left\|\frac{\partial e}{\partial t}\right\|+c_{2}\|e\|+c_{3}+c_{4}\left\|\frac{\partial e}{\partial t}\right\|\|\tilde{a}\|+c_{5}\left\|\frac{\partial e}{\partial t}\right\|\|\tilde{\sigma}\|
$$

where $\left\{c_{i}\right\}_{i=1}^{3}$ are defined in Corollary $1, c_{4}=\left\|\Phi^{\top} \gamma^{\top}\right\|$, and $c_{5}=\|\varphi\|$.

We now propose the following adaptive laws for $\hat{a}(t)$ and $\hat{\sigma}(t)$. 
Theorem 4: The following adaptive laws results in bounded tracking of the closed-loop uncertain Lagrangian system (W).

$$
\begin{aligned}
& \dot{\hat{a}}=\Gamma_{a} \int_{\Omega} \gamma \Phi \frac{\partial e}{\partial t} d x \\
& \dot{\hat{\sigma}}=\Gamma_{\sigma} \int_{\Omega} \varphi^{\top} \frac{\partial e}{\partial t} d x
\end{aligned}
$$

where $\Gamma_{a}>0 \in \mathbb{R}^{r \times r}$ and $\Gamma_{\sigma}>0 \in \mathbb{R}^{N \times N}$ are the adaptive gain or learning rate matrices.

Proof: We choose a Lyapunov function

$$
V=H+\frac{1}{2} \tilde{a}^{\top} \Gamma_{a}^{-1} \tilde{a}+\frac{1}{2} \tilde{\sigma}^{\top} \Gamma_{\sigma}^{-1} \tilde{\sigma}
$$

Then, $\dot{V}(t)$ is evaluated as

$$
\begin{aligned}
\dot{V} \leq-\int_{\Omega} \frac{\partial e}{\partial t} \cdot\left[m(x) c \frac{\partial e}{\partial t}+\Phi^{\top} \gamma^{\top} \tilde{a}+\varphi \tilde{\sigma}\right] d x+\tilde{a}^{\top} & \Gamma_{a}^{-1} \dot{\tilde{a}}+\tilde{\sigma}^{\top} \Gamma_{\sigma}^{-1} \dot{\tilde{\sigma}}+\lambda_{\max }\left(\Gamma_{\sigma}^{-1}\right) \delta\|\tilde{\sigma}\| \\
& +c_{1}\left\|\frac{\partial e}{\partial t}\right\|+c_{2}\|e\|+c_{3}+c_{4}\left\|\frac{\partial e}{\partial t}\right\|\|\tilde{a}\|+c_{5}\left\|\frac{\partial e}{\partial t}\right\|\|\tilde{\sigma}\|
\end{aligned}
$$

which can also be expressed as

$$
\begin{aligned}
\dot{V} \leq-\int_{\Omega} \frac{\partial e^{\top}}{\partial t} m(x) c \frac{\partial e}{\partial t} d x+\tilde{a}^{\top} & \left(\Gamma_{a}^{-1} \dot{\tilde{a}}-\int_{\Omega} \gamma \Phi \frac{\partial e}{\partial t} d x\right)+\tilde{\sigma}^{\top}\left(\Gamma_{\sigma}^{-1} \dot{\tilde{\sigma}}-\int_{\Omega} \varphi^{\top} \frac{\partial e}{\partial t} d x\right) \\
& +\lambda_{\max }\left(\Gamma_{\sigma}^{-1}\right) \delta\|\tilde{\sigma}\|+c_{1}\left\|\frac{\partial e}{\partial t}\right\|+c_{2}\|e\|+c_{3}+c_{4}\left\|\frac{\partial e}{\partial t}\right\|\|\tilde{a}\|+c_{5}\left\|\frac{\partial e}{\partial t}\right\|\|\tilde{\sigma}\|
\end{aligned}
$$

Setting the second and third terms on the right hand side of the inequality yields the adaptive laws.

Thus, $\dot{V} \leq 0$ if $\left\|\frac{\partial e}{\partial t}\right\| \geq c_{6}$ where $c_{6}$ is some positive small number. Therefore, the tracking error of the closed-loop uncertain Lagrangian system (W) is bounded.

Note that the adaptive laws can be made to be ultimately bounded by adding a robust modification term such as the $\sigma$-modification ${ }^{4}$ or the optimal control modification. ${ }^{11,12}$ We state without proof the optimal control modification adaptive laws

$$
\begin{gathered}
\dot{\hat{a}}=\Gamma_{a}\left(\int_{\Omega} \gamma \Phi \frac{\partial e}{\partial t} d x-v \int_{\Omega} \gamma \Phi \Phi^{\top} \gamma^{\top} d x \hat{a}\right) \\
\dot{\hat{\sigma}}=\Gamma_{\sigma}\left(\int_{\Omega} \varphi^{\top} \frac{\partial e}{\partial t} d x-v \int_{\Omega} \varphi^{\top} \varphi d x \hat{\sigma}\right)
\end{gathered}
$$

\section{Adaptive Flutter Suppression Control Application}

Consider a general bending-torsion motion of a swept wing with an elastic axis sweep angle of $\Lambda$. The aeroelastic equations of motion are described by ${ }^{10}$

$$
\begin{gathered}
\rho A \frac{\partial^{2} z}{\partial t^{2}}+\rho A e_{c g} \frac{\partial^{2} \theta}{\partial t^{2}}+\frac{\partial^{2}}{\partial x^{2}}\left(E I \frac{\partial^{2} z}{\partial x^{2}}\right)=\bar{l} \cos \Lambda+\frac{\partial \bar{m}}{\partial x} \cos \Lambda \sin \Lambda+l_{\delta} u \\
\rho I_{x x} \frac{\partial^{2} \theta}{\partial t^{2}}+\rho A e_{c g} \frac{\partial^{2} z}{\partial t^{2}}-\frac{\partial}{\partial x}\left(G J \frac{\partial \theta}{\partial x}\right)=\bar{m} \cos ^{2} \Lambda+m_{\delta} u
\end{gathered}
$$

where $z(x, t)$ is the bending displacement and $\theta(x, t)$ is the torsional rotation. The right hand sides of the aeroelastic equations are the aerodynamic force $\bar{l}(x, t)$ and moment $\bar{m}(x, t)$ and control force $l_{\delta}(x, t) u(t)$ and moment $m_{\delta}(x, t) u(t)$. The control input vector $u(t)=\left[\begin{array}{lll}\delta_{1}(t) & \cdots & \delta_{p}(t)\end{array}\right]^{\top} \in \mathbb{R}^{p}$ comprises $p$ discrete control surface deflections that span the wing over the intervals $\Omega_{c_{i}}=\left(a_{c_{i}}, b_{c_{i}}\right), i=1, \ldots, p$ where $0<a_{c_{i}}<x<b_{c_{i}}<L$ and $L$ is the 
length of the wing. Figure 1 illustrates several discrete control surfaces of a flexible wing. The sensors are two wing shape sensors, illustrated as two red dashed lines, that can produce the vertical displacements $z_{1}(x, t)$ and $z_{2}(x, t)$ as well as their velocities. The bending displacement $z(x, t)$ and torsional rotation $\theta(x, t)$ can be directly computed from these measurements. To illustrate this, we consider the placements of the forward and aft wing shape sensors to be at distances of $e_{1}$ and $e_{2}$ from the elastic axis, respectively. We can estimate $z(x, t)$ and $\theta(x, t)$ by a linear interpolation as

$$
\begin{gathered}
z=z_{1}+\frac{z_{2}-z_{1}}{1+\frac{e_{2}}{e_{1}}} \\
\theta=\frac{z_{1}-z_{2}}{e_{1}+e_{2}}
\end{gathered}
$$

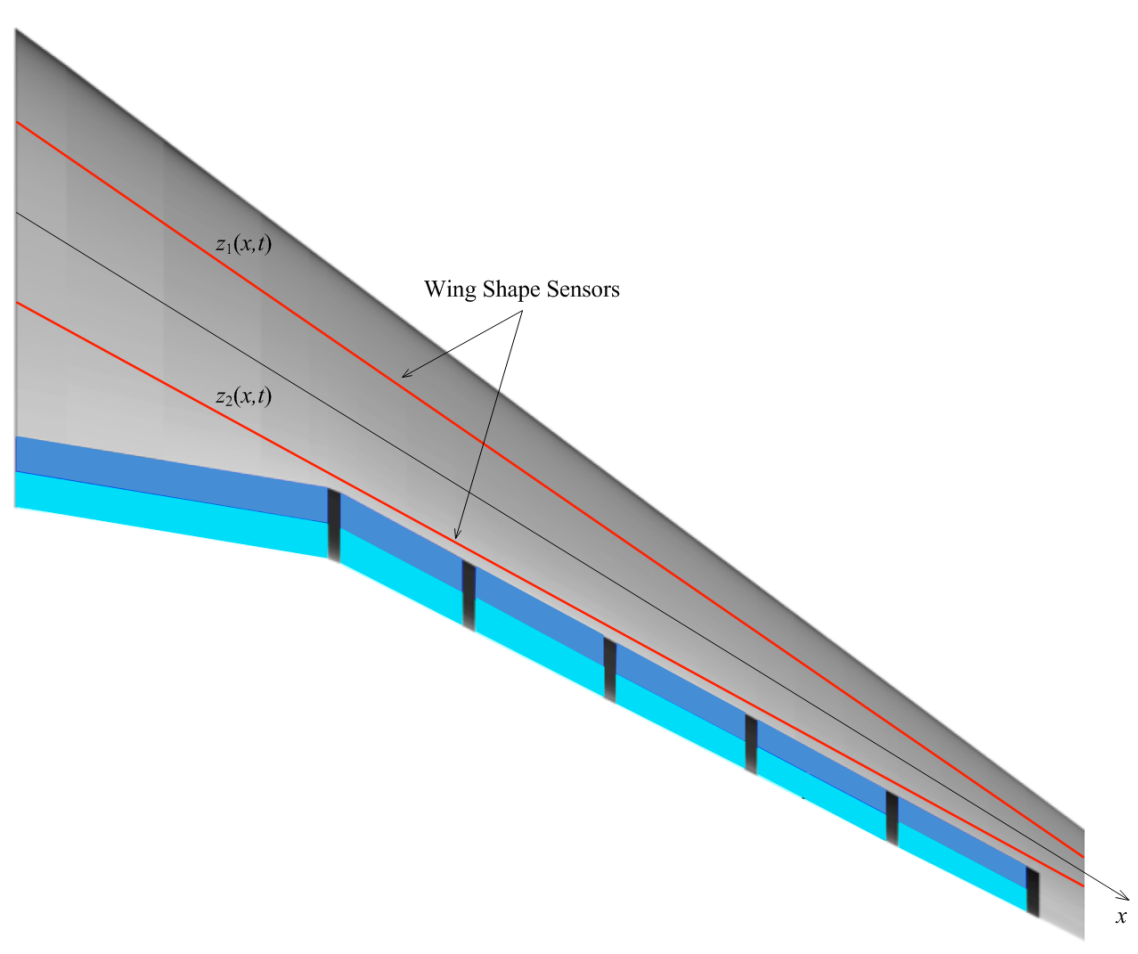

Figure 1. Discrete Control Surfaces of a Flexible Wing with Continuous Wing Shape Sensors

The unsteady lift force $\bar{l}(x, t)$ and pitching moment $\bar{m}(x, t)$ in the streamwise direction are given by

$$
\begin{aligned}
& \bar{l}=\bar{l}_{r}+C(k) c_{L_{\alpha}} q_{\infty} c\left(\theta \cos \Lambda-\frac{\partial z}{\partial x} \sin \Lambda+\frac{e_{c}}{V_{\infty}} \frac{\partial \theta}{\partial t} \cos \Lambda\right.\left.-\frac{1}{V_{\infty}} \frac{\partial z}{\partial t}\right) \\
&+\frac{\pi \rho_{\infty} V_{\infty} c^{2}}{4}\left(\frac{\partial \theta}{\partial t} \cos \Lambda+\frac{e_{m}}{V_{\infty}} \frac{\partial^{2} \theta}{\partial t^{2}} \cos \Lambda-\frac{1}{V_{\infty}} \frac{\partial^{2} z}{\partial t^{2}}\right) \\
& \bar{m}=\bar{m}_{r}+C(k) c_{L_{\alpha}} q_{\infty} c e(\left.\theta \cos \Lambda-\frac{\partial z}{\partial x} \sin \Lambda+\frac{e_{c}}{V_{\infty}} \frac{\partial \theta}{\partial t} \cos \Lambda-\frac{1}{V_{\infty}} \frac{\partial z}{\partial t}\right) \\
&-\frac{\pi \rho_{\infty} c^{2} e_{m}}{4}\left(e_{m} \frac{\partial^{2} \theta}{\partial t^{2}} \cos \Lambda-\frac{\partial^{2} z}{\partial t^{2}}\right)-\frac{\pi \rho_{\infty} V_{\infty} c^{2} e_{c}}{4} \frac{\partial \theta}{\partial t} \cos \Lambda-\frac{\pi \rho_{\infty} c^{4}}{128} \frac{\partial^{2} \theta}{\partial t^{2}} \cos \Lambda
\end{aligned}
$$

The distributed control force and moment sensitivities are represented by $l_{\delta}(x)$ and $m_{\delta}(x)$, respectively. The kinetic energy density of the wing is expressed as

$$
\tau=\frac{1}{2} \frac{\partial w^{\top}}{\partial t}\left[m(x)+m_{a}(x)\right] \frac{\partial w}{\partial t}
$$


where $w(x, t)=\left[\begin{array}{ll}z(x, t) & \theta(x, t)\end{array}\right]^{\top}$. The mass matrices $m(x)$ and $m_{a}(x)$ are given by

$$
\begin{gathered}
m(x)=\rho A\left[\begin{array}{cc}
1 & e_{c g} \\
e_{c g} & \frac{I}{A}
\end{array}\right] \\
m_{a}(x)=\frac{\pi \rho_{\infty} c^{2} \cos \Lambda}{4}\left[\begin{array}{cc}
1 & -e_{m} \cos \Lambda \\
-e_{m} \cos \Lambda & \left(e_{m}^{2}+\frac{c^{2}}{32}\right) \cos ^{2} \Lambda
\end{array}\right]
\end{gathered}
$$

where $m(x)$ is the structural mass matrix and $m_{a}(x)$ is the aerodynamic mass matrix due to the so-called apparent mass effect in aeroelasticity.

The potential energy density of the wing is expressed as

$$
v=\frac{1}{2} \frac{\partial w^{\top}}{\partial x} k_{1}(x) \frac{\partial w}{\partial x}+\frac{1}{2} \frac{\partial^{2} w^{\top}}{\partial x^{2}} k_{2}(x) \frac{\partial^{2} w}{\partial x^{2}}
$$

where

$$
\begin{aligned}
& k_{1}(x)=\left[\begin{array}{cc}
0 & 0 \\
0 & G J
\end{array}\right] \\
& k_{2}(x)=\left[\begin{array}{cc}
E I & 0 \\
0 & 0
\end{array}\right]
\end{aligned}
$$

and $E I(x)$ and $G J(x)$ are the bending and torsional stiffness properties of the wing structure.

The Lagrangian density is obtained as

$$
\mathscr{L}=\tau-v=\frac{1}{2} \frac{\partial w^{\top}}{\partial t}\left[m(x)+m_{a}(x)\right] \frac{\partial w}{\partial t}-\frac{1}{2} \frac{\partial w^{\top}}{\partial x} k_{1}(x) \frac{\partial w}{\partial x}-\frac{1}{2} \frac{\partial^{2} w^{\top}}{\partial x^{2}} k_{2}(x) \frac{\partial^{2} w}{\partial x^{2}}
$$

The linear operator $L$ is given by

$$
L=m^{-1}(x)\left[-\frac{\partial}{\partial x}\left(k_{1}(x) \frac{\partial}{\partial x}\right)+\frac{\partial^{2}}{\partial x^{2}}\left(k_{2}(x) \frac{\partial^{2}}{\partial x^{2}}\right)\right]
$$

The Euler-Lagrange equation in vector form is expressed as

$$
\frac{\partial}{\partial t}\left(\frac{\partial \mathscr{L}}{\partial\left(\frac{\partial W}{\partial t}^{\top}\right)}\right)+\frac{\partial}{\partial x}\left(\frac{\partial \mathscr{L}}{\partial\left(\frac{\partial W}{\partial x}^{\top}\right)}\right)-\frac{\partial^{2}}{\partial x^{2}}\left(\frac{\partial \mathscr{L}}{\partial\left(\frac{\partial^{2} w}{\partial x^{2}}\right)}\right)=q_{c}+b u
$$

Evaluating the Euler-Lagrange equation yields a system of a $2^{\text {nd }}$-order PDE and $4^{\text {th }}$-order PDE

$$
\left[m(x)+m_{a}(x)\right] \frac{\partial^{2} w}{\partial t^{2}}-\frac{\partial}{\partial x}\left(k_{1}(x) \frac{\partial w}{\partial x}\right)+\frac{\partial^{2}}{\partial x^{2}}\left(k_{2}(x) \frac{\partial^{2} w}{\partial x^{2}}\right)=q_{c}+b u
$$

The anti-symmetric boundary conditions of the wing are $w(0, t)=0,\left.E I \frac{\partial^{2} z}{\partial x^{2}}\right|_{x=L}=0,\left.\frac{\partial}{\partial x}\left(E I \frac{\partial^{2} z}{\partial x^{2}}\right)\right|_{x=L}=0$, and $\left.G J \frac{\partial \theta}{\partial x}\right|_{x=L}=0$. These boundary conditions satisfy the compact support of the distributed system in Eq. (123). as

The non-conservative force vector $q_{c}$ due to the unsteady lift force $\bar{l}(x, t)$ and pitching moment $\bar{m}(x, t)$ is expressed

$$
q_{c}=C(k)\left(q_{1} w+q_{2} \frac{\partial w}{\partial x}+q_{3} \frac{\partial w}{\partial t}\right)+q_{4} \frac{\partial w}{\partial t}+f
$$

where $C(k)$ is the Theodorsen's function, $k=\frac{\omega c}{2 V_{\infty}}$ is the reduced frequency, and $\left\{q_{i}\right\}_{i=1}^{4}$ are given by

$$
q_{1}=c_{L_{\alpha}} q_{\infty} c \cos ^{2} \Lambda\left[\begin{array}{cc}
0 & 1 \\
0 & e \cos \Lambda
\end{array}\right]
$$




$$
\begin{gathered}
q_{2}=c_{L_{\alpha}} q_{\infty} c \cos ^{2} \Lambda\left[\begin{array}{cc}
-\tan \Lambda & 0 \\
-e \sin \Lambda & 0
\end{array}\right] \\
q_{3}=\frac{c_{L_{\alpha}} \rho_{\infty} V_{\infty} c \cos \Lambda}{2}\left[\begin{array}{cc}
-1 & e_{c} \cos \Lambda \\
-e \cos \Lambda & e e_{c} \cos ^{2} \Lambda
\end{array}\right] \\
q_{4}=\frac{\pi \rho_{\infty} V_{\infty} c^{2} \cos ^{2} \Lambda}{4}\left[\begin{array}{cc}
0 & 1 \\
0 & -e_{c} \cos \Lambda
\end{array}\right]
\end{gathered}
$$

The pitching moment contribution to the lift force due to the wing sweep is represented by $f$ which is given by

$$
f=\left[\begin{array}{c}
\bar{m} \cos \Lambda \sin \Lambda \\
0
\end{array}\right]
$$

The Theodorsen's function $C(k)=F(k)+i G(k)$ operates on an harmonic input $y(t)=y_{0} e^{i \omega t}$ at a constant frequency $\omega$ as

$$
C(k) y=F(k) y+i G(k) y=F(k) y+\frac{G(k) c \dot{y}}{2 k V_{\infty}}
$$

The distributed control sensitivity $b(x)$ due to the distributed control force and moment sensitivities $l_{\delta}(x)$ and $m_{\delta}(x)$ is expressed as

$$
b=C(k) q_{5}+\sum_{i=1}^{n} q_{6, i}
$$

where

$$
\begin{gathered}
q_{5}=q_{\infty} c \cos ^{2} \Lambda\left[\begin{array}{c}
c_{L_{\delta}} \\
c_{L_{\delta}} e \cos \Lambda
\end{array}\right] \\
q_{6}=q_{\infty} c^{2} \cos ^{3} \Lambda\left[\begin{array}{c}
0 \\
\bar{c}_{m_{\delta}}
\end{array}\right]
\end{gathered}
$$

The uncertainty is assumed to exist in the value of $c_{L_{\alpha}}(x)$ and in the form of a control uncertainty due the control surface "buzzing" as the wing approaches flutter. It is represented by

$$
\Delta q_{c}=C(k) \Delta c_{L_{\alpha}}\left(\frac{q_{1}}{c_{L_{\alpha}}} w+\frac{q_{2}}{c_{L_{\alpha}}} \frac{\partial w}{\partial x}+\frac{q_{3}}{c_{L_{\alpha}}} \frac{\partial w}{\partial t}\right)+b(x) \Delta u
$$

where $\Delta c_{L_{\alpha}}(x)$ and $\Delta u(t)$ are unknown. We can parameterize $\Delta c_{L_{\alpha}}(x)$ as a cubic polynomial as

$$
\Delta c_{L_{\alpha}}=\gamma^{\top} a=\sum_{i=0}^{3} a_{i} x^{i}
$$

Then, the uncertainty is expressed as shown in Eq. (94) with the parametric uncertainty $a=\left[\begin{array}{llll}a_{3} & a_{2} & a_{1} & a_{0}\end{array}\right]^{\top}$

$$
\Delta q_{c}=\Phi^{\top} \gamma^{\top} a
$$

where

$$
\begin{gathered}
\Phi^{\top}=C(k)\left[\begin{array}{ccc}
\frac{q_{1}}{c_{L_{\alpha}}} w & \frac{q_{2}}{c_{L_{\alpha}}} \frac{\partial w}{\partial x} & \frac{q_{3}}{c_{L_{\alpha}}} \frac{\partial w}{\partial t}
\end{array}\right] \\
\gamma^{\top}=\left[\begin{array}{llll}
x^{3} & x^{2} & x & 1 \\
x^{3} & x^{2} & x & 1 \\
x^{3} & x^{2} & x & 1
\end{array}\right]
\end{gathered}
$$


The nominal discrete control is given by

$$
u=-B^{\top}\left(B B^{\top}\right)^{-1} \int_{\Omega} \varphi^{\top}\left[q_{c}+m(x) c \frac{\partial w}{\partial t}+\mu \frac{\partial}{\partial x}\left(k_{1}(x) \frac{\partial w}{\partial x}\right)-\mu \frac{\partial^{2}}{\partial x^{2}}\left(k_{2}(x) \frac{\partial^{2} w}{\partial x^{2}}\right)\right] d x
$$

Integrating by parts the last two terms yields

$$
u=-B^{\top}\left(B B^{\top}\right)^{-1} \int_{\Omega}\left[\varphi^{\top} q_{c}+\varphi^{\top} m(x) c \frac{\partial w}{\partial t}-\frac{\partial \varphi^{\top}}{\partial x} \mu k_{1}(x) \frac{\partial w}{\partial x}-\frac{\partial^{2} \varphi^{\top}}{\partial x^{2}} \mu k_{2}(x) \frac{\partial^{2} w}{\partial x^{2}}\right] d x
$$

In addition, we also consider the strong-form discrete control in the form

$$
u=-B^{\top}\left(B B^{\top}\right)^{-1} \int_{\Omega}\left[\frac{\partial w}{\partial t} \cdot q_{c}+\frac{\partial w}{\partial t} \cdot m(x) c \frac{\partial w}{\partial t}-\frac{\partial^{2} w}{\partial x \partial t} \cdot \mu k_{1}(x) \frac{\partial w}{\partial x}-\frac{\partial^{3} w}{\partial x^{2} \partial t} \cdot \mu k_{2}(x) \frac{\partial^{2} w}{\partial x^{2}}\right] d x
$$

The adaptive augmentation control is designed as

$$
u_{a d}=-B^{\top}\left(B B^{\top}\right)^{-1} \int_{\Omega} \varphi^{\top}\left(\Phi^{\top} \gamma^{\top} \hat{a}+b \Delta \hat{u}\right) d x
$$

where $\Delta \hat{u}(t)$ is estimated by the following adaptive law:

$$
\Delta \dot{\hat{u}}=\Gamma_{\Delta u} \int_{\Omega} b^{\top} \frac{\partial e}{\partial t} d x
$$

A numerical simulation is performed. The wing has a length $L=80 \mathrm{ft}$ and five control surfaces of equal length of $0.2 \mathrm{~L}$. A flutter analysis is conducted for the first four symmetric modes in bending and torsion. Figures 2 and 3 show the frequencies $\omega$ and structural damping values $g$ as functions of the airspeed $V_{\infty}$. A flutter corresponding to the second torsion mode $2 \mathrm{~T}$ at a frequency of $50.64 \mathrm{rad} / \mathrm{sec}$ occurs at an airspeed of $376 \mathrm{ft} / \mathrm{sec}$ when $g$ crosses zero. The system is unstable at and above $376 \mathrm{ft} / \mathrm{sec}$.

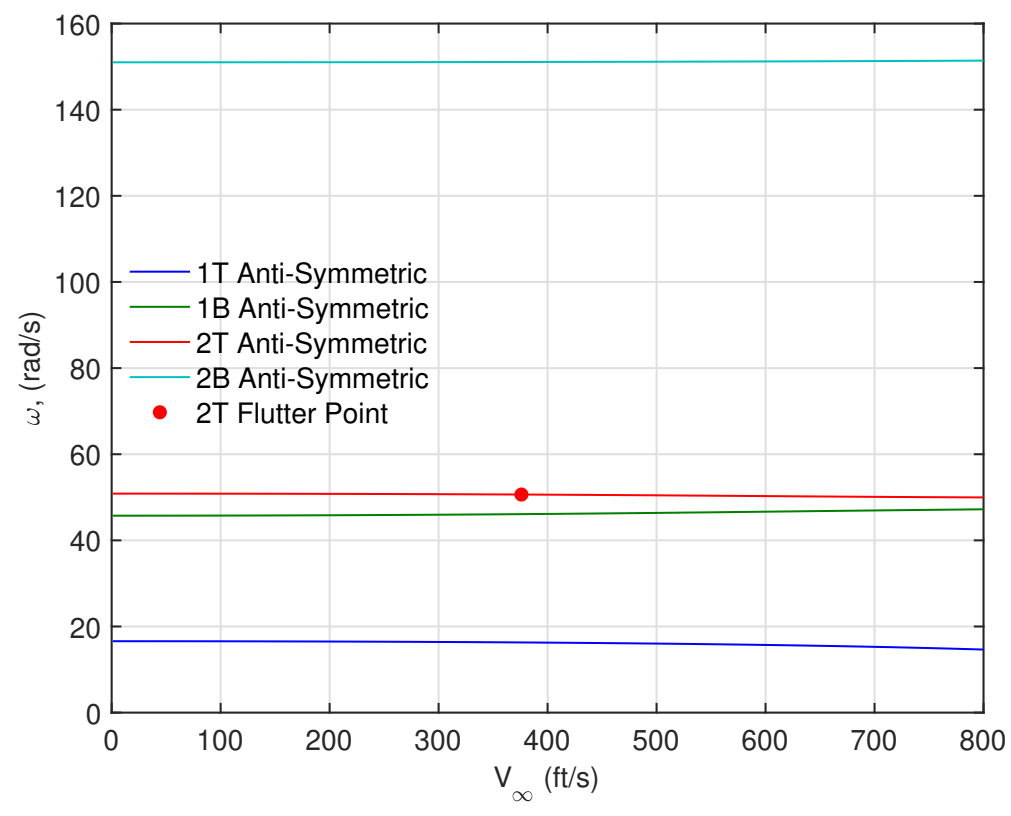

Figure 2. $V-\omega$ Diagram of Frequencies of Anti-Symmetric Modes 


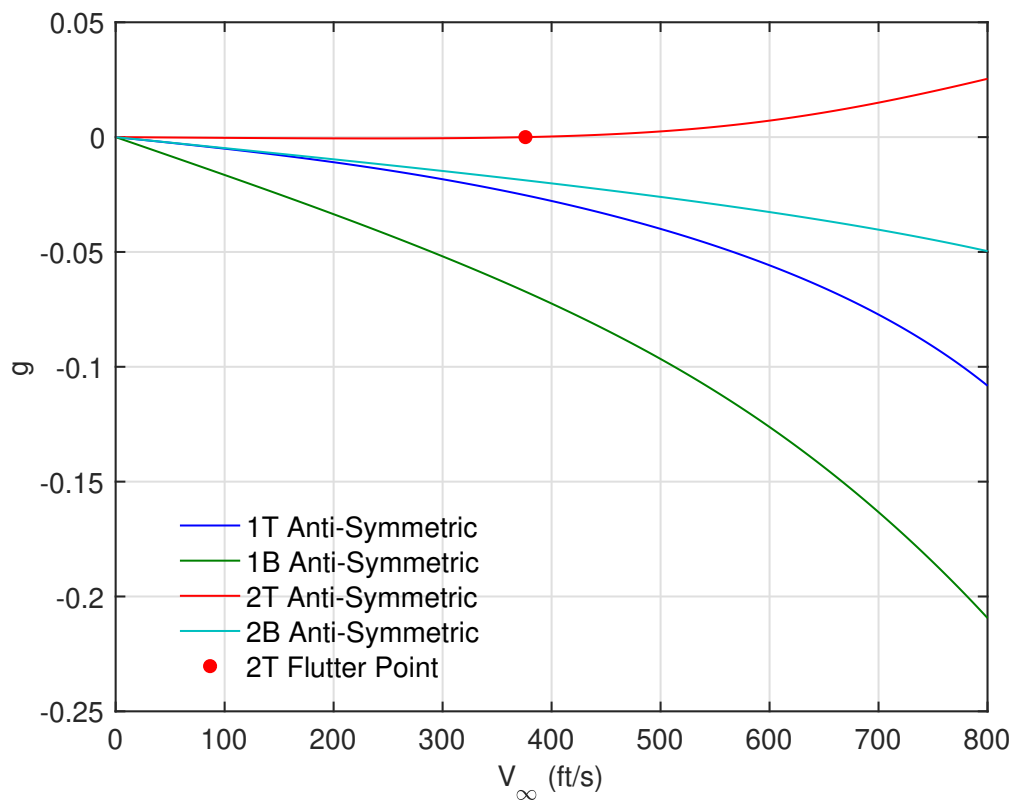

Figure 3. $V-g$ Diagram of Damping of Anti-Symmetric Modes

Figures 4 and 5 show the nominal open-loop response of the wing in bending and torsion at $400 \mathrm{ft} / \mathrm{sec}$ just beyond the flutter point without flutter suppression control and uncertainty in the first 10 seconds. As can be seen, the motion of the wing in bending and torsion is slowly divergent.

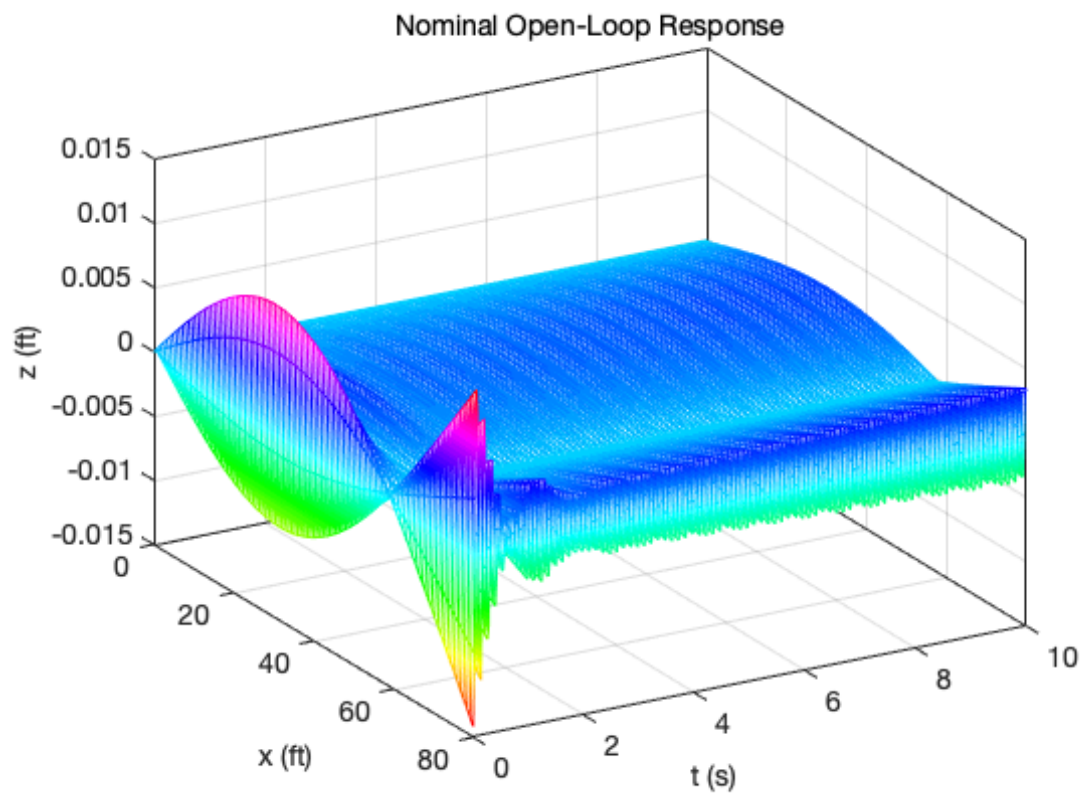

Figure 4. Wing Bending Deflection $z(x, t)$ without Flutter Suppression Control and Uncertainty 


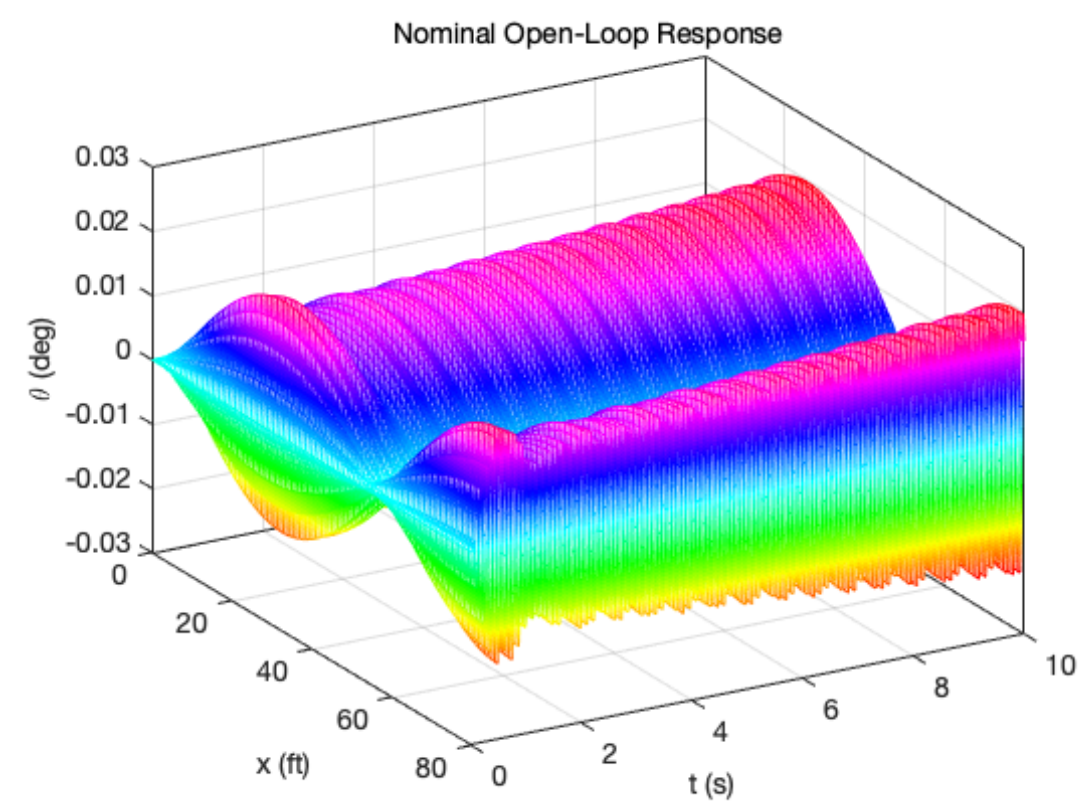

Figure 5. Wing Torsional Twist $\theta(x, t)$ without Flutter Suppression Control and Uncertainty
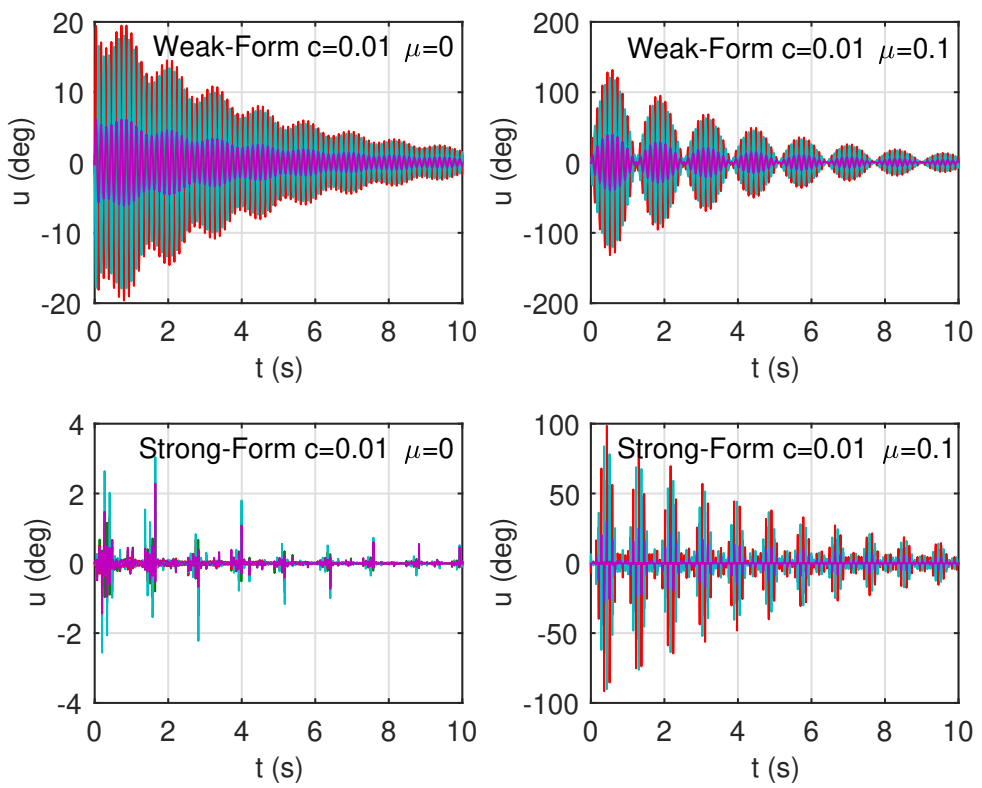

Figure 6. Comparison of Nominal Flutter Suppression Controllers $u(t)$

A nominal flutter suppression control is designed using the weak-form and strong-form discrete controls from Eq. (80) and (81), respectively. The displacement and velocity feedback gains are selected to be $\mu=0.1$ and $c=0.01 \omega_{f}$, respectively, where $\omega_{f}$ is the flutter frequency. Figure 6 is the plot of the control surface deflections for the four nominal controllers. No saturation limit is applied in the control simulations for the purpose of comparison. The linear weak-form controllers produce much larger control surface deflections than the nonlinear strong-form controllers. The strong-form controller with $\mu=0$ produces the smallest control surface deflections, thus demonstrating the result of 
Theorem 3. Increasing or decreasing $\mu$ has an effect of increasing the control amplitude, so the minimum control amplitude corresponds to $\mu=0$.

The Hamiltonians for the four nominal controllers are plotted in Fig. 7 and are quite similar. The strong-form controller with $\mu=0.1$ produces the largest decrease of the Hamiltonian, thus is more effective, but by the same token requires more control power which clearly can be seen from Fig. 6 . The strong-form controller with $\mu=0$ requires the least amount of control power and therefore produces a smaller decrease in the Hamiltonian. This is in agreement with the result of Theorem 3 .

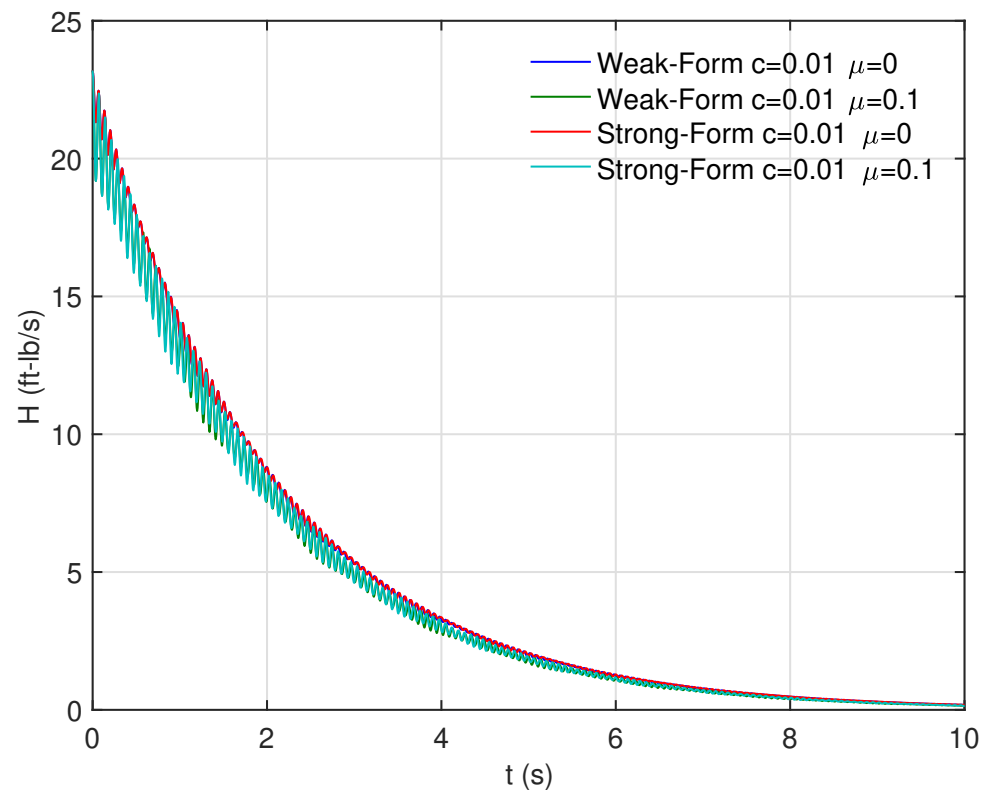

Figure 7. Hamiltonian Function $H(t)$ for Nominal Flutter Suppression Controllers

Figures 8 and 9 show the nominal closed-loop response of the wing in bending and torsion at $800 \mathrm{ft} / \mathrm{sec}$ with the weak-form flutter suppression controller with $c=0.01$ and $\mu=0$. It can be seen that the flutter suppression control based on the Hamilton's principle is able to stabilize the wing motion. For comparison, Figures 10 and 11 show the nominal closed-loop response of the wing with the strong-form flutter suppression controller with $c=0.01$ and $\mu=0$. The nominal closed-loop response with the strong-form controller is very similar to that with the weak-form controller, but the response is produced by the much smaller control surface deflections with the strong-form controller than those with the weak-form controller. 


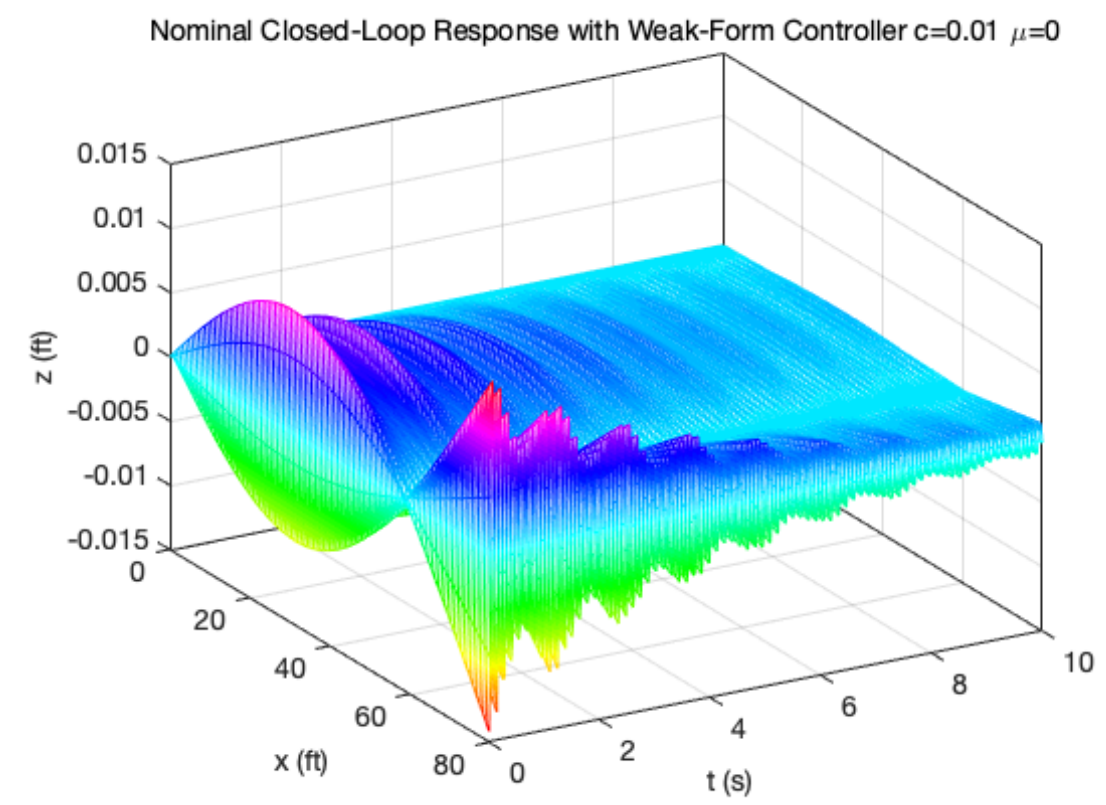

Figure 8. Wing Bending Deflection $z(x, t)$ with Weak-Form Flutter Suppression Controller $c=0.01 \mu=0$

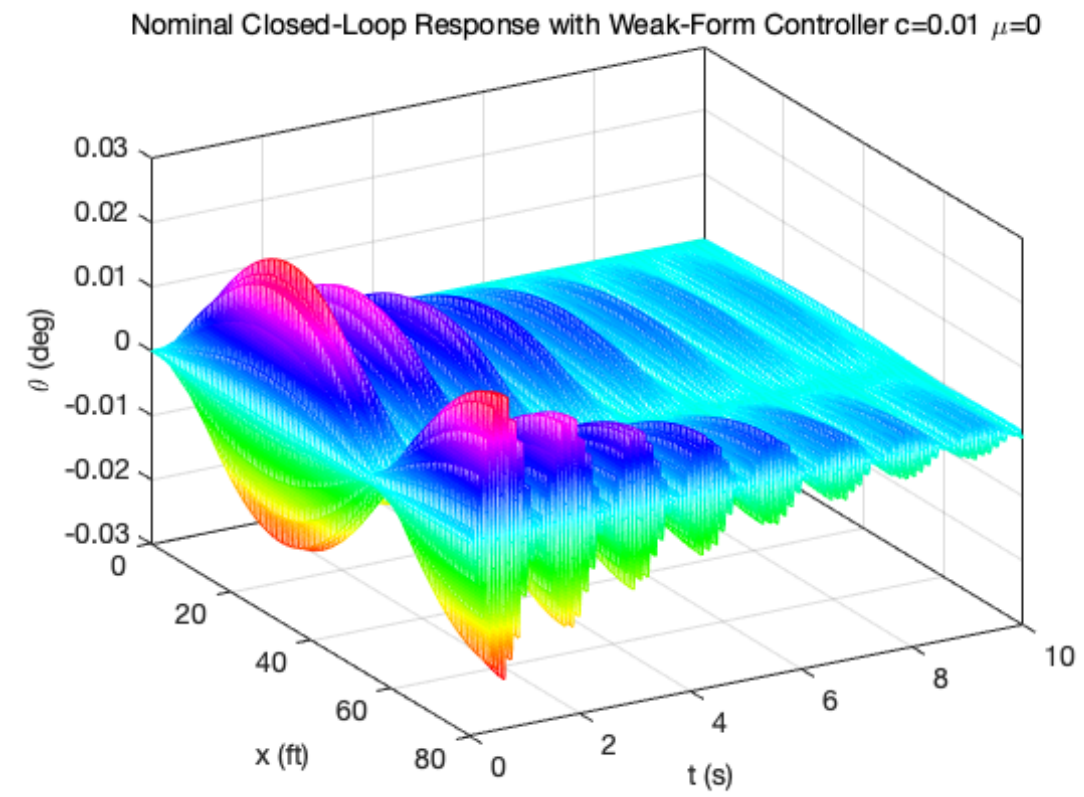

Figure 9. Wing Torsional Twist $\theta(x, t)$ with Weak-Form Flutter Suppression Controller $c=0.01 \mu=0$ 


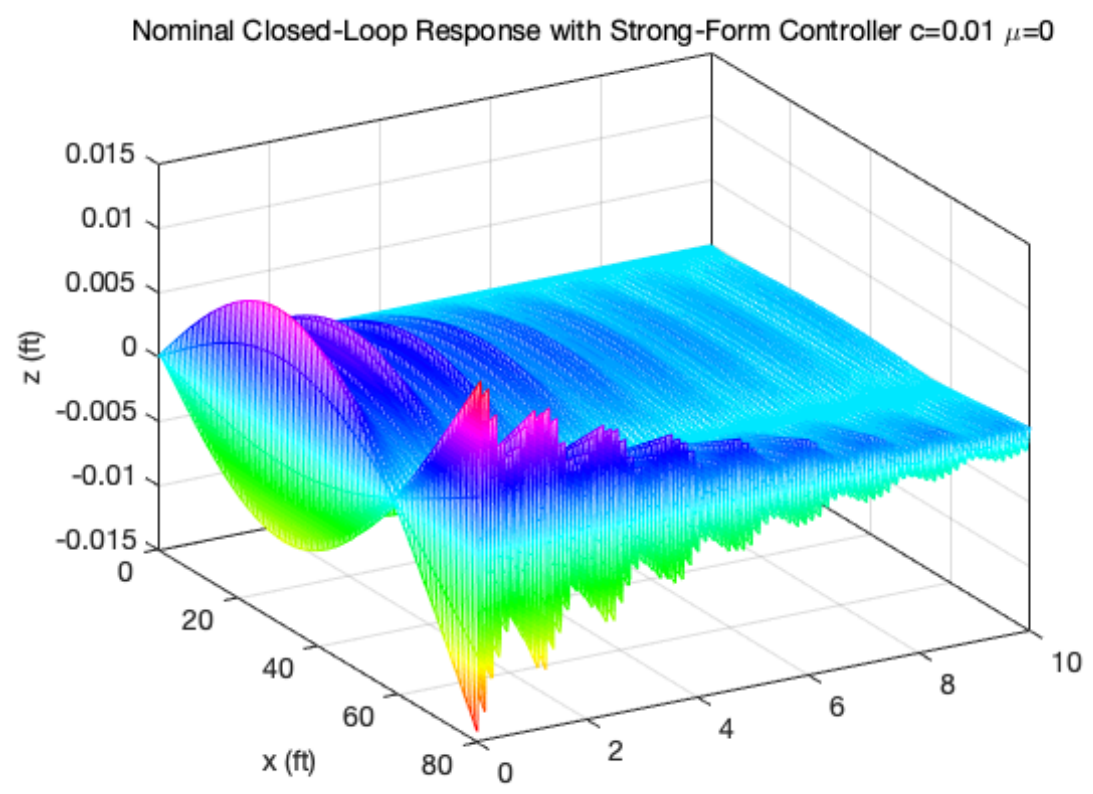

Figure 10. Wing Bending Deflection $z(x, t)$ with Strong-Form Flutter Suppression Controller $c=0.01 \mu=0$

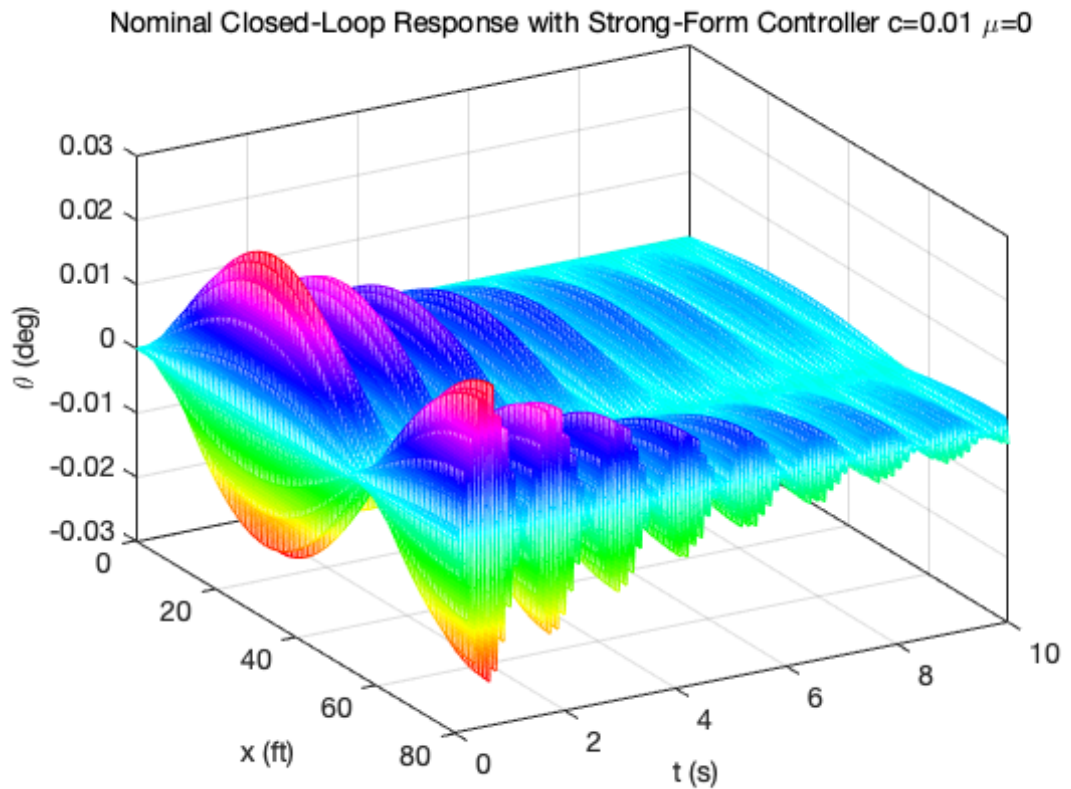

Figure 11. Wing Torsional Twist $\theta(x, t)$ with Strong-Form Flutter Suppression Controller $c=0.01 \mu=0$

To illustrate the adaptive control design using the Hamilton's principle, we conduct a simulation with the uncertainty. We choose the parametric uncertainty $a=\left[\begin{array}{llll}-1.125 & 0.9 & 0.125 & 0.3\end{array}\right]^{\top}$. The control surface "buzzing" uncertainty is modeled by $\Delta u(t)=\Delta u_{0} \sin \omega_{f} t$ where $\Delta u_{0}=1^{\circ}$. The reference model is chosen to be the nominal closed-loop plant with the nominal weak-form flutter suppression controller, shown in Figs. 8 and 9. The adaptive laws are implemented using $\Gamma_{a}=100 I$ and $\Gamma_{\Delta u}=10$. Figure 12 shows the time history of the estimate $\hat{a}(t)$. As can be seen, the estimate $\hat{a}(t)$ tends to a constant set of values but does not converge to the true parametric uncertainty $a$. 
This is not a surprising result since model-reference adaptive control does not guarantee any parameter convergence.

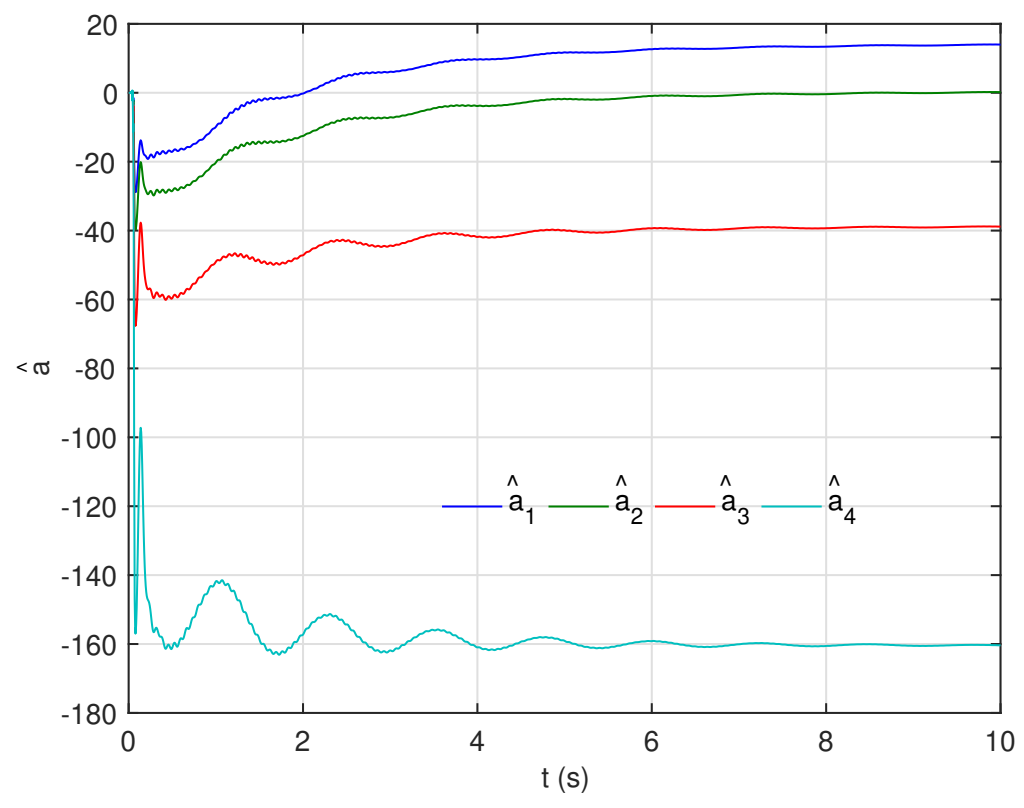

Figure 12. Estimate of Parametric Uncertainty $\hat{a}(t)$

Figures 13 and 14 show the wing motion without adaptive control. The control surface "buzzing" uncertainty causes the wing motion, which is previously stable with the nominal flutter suppression controller, to begin to diverge. The adaptive controller is able to regain stability as shown in Figures 15 and 16. Thus, the effectiveness of modelreference adaptive control based on the Hamilton's principle is demonstrated.

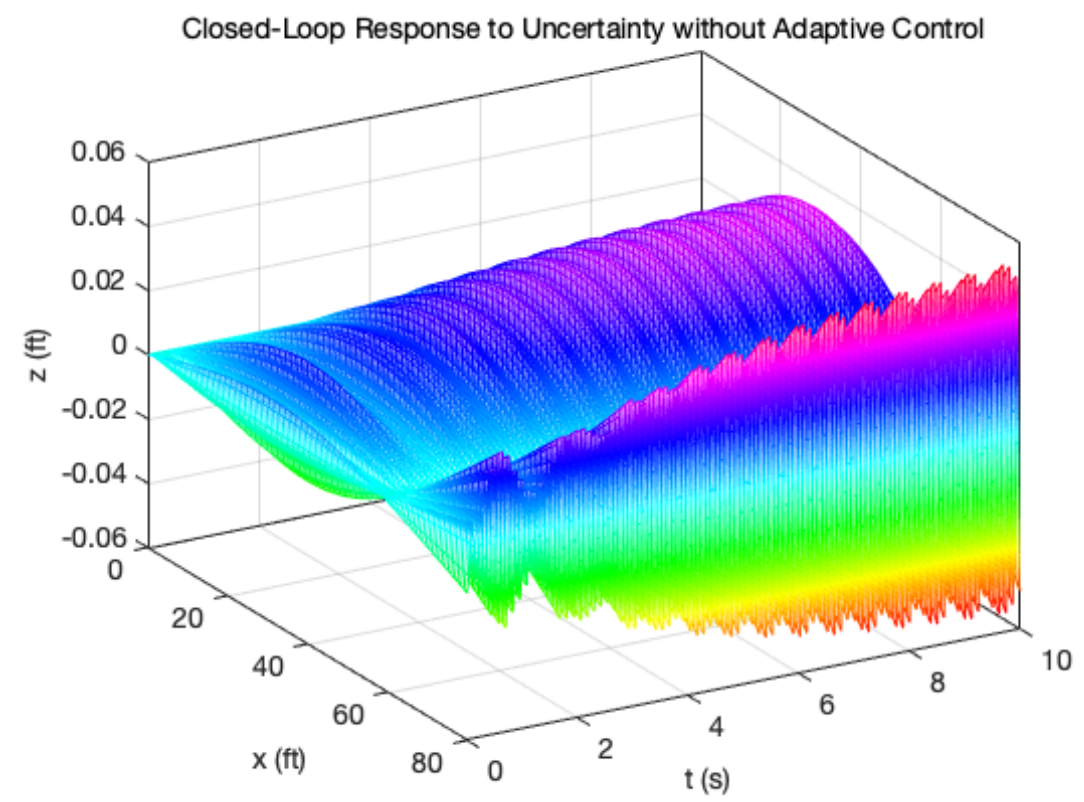

Figure 13. Wing Bending Deflection $z(x, t)$ without Adaptive Control 


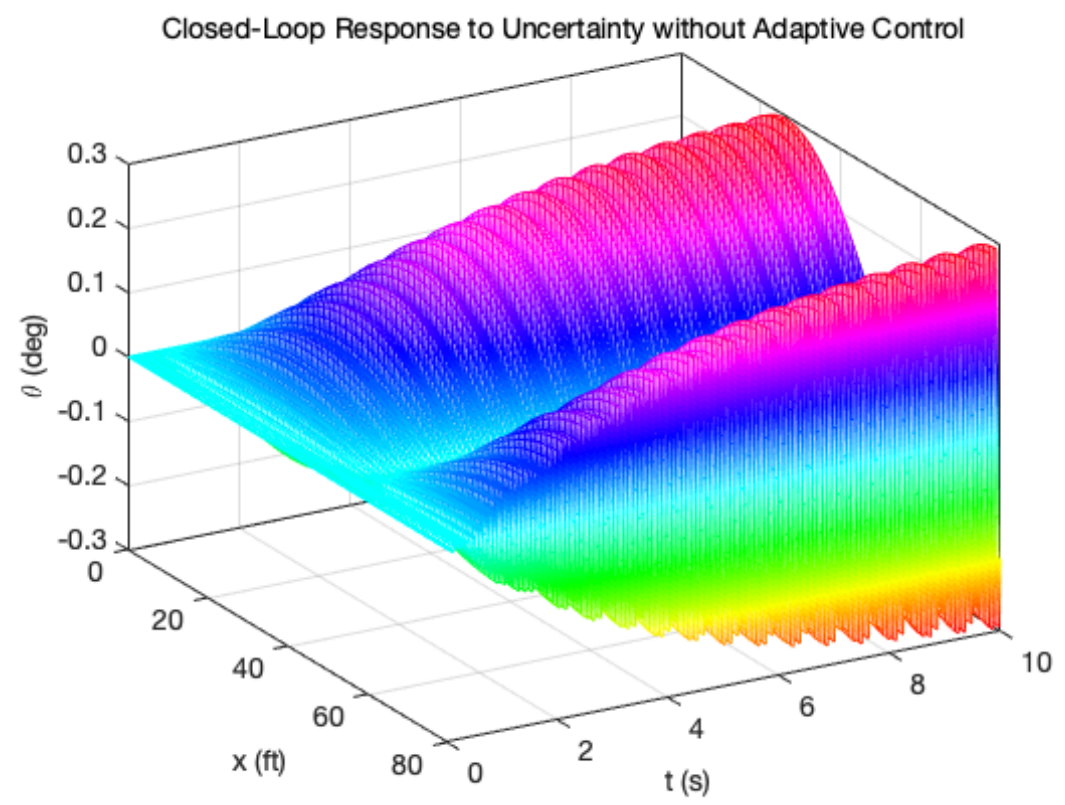

Figure 14. Wing Torsional Twist $\theta(x, t)$ without Adaptive Control

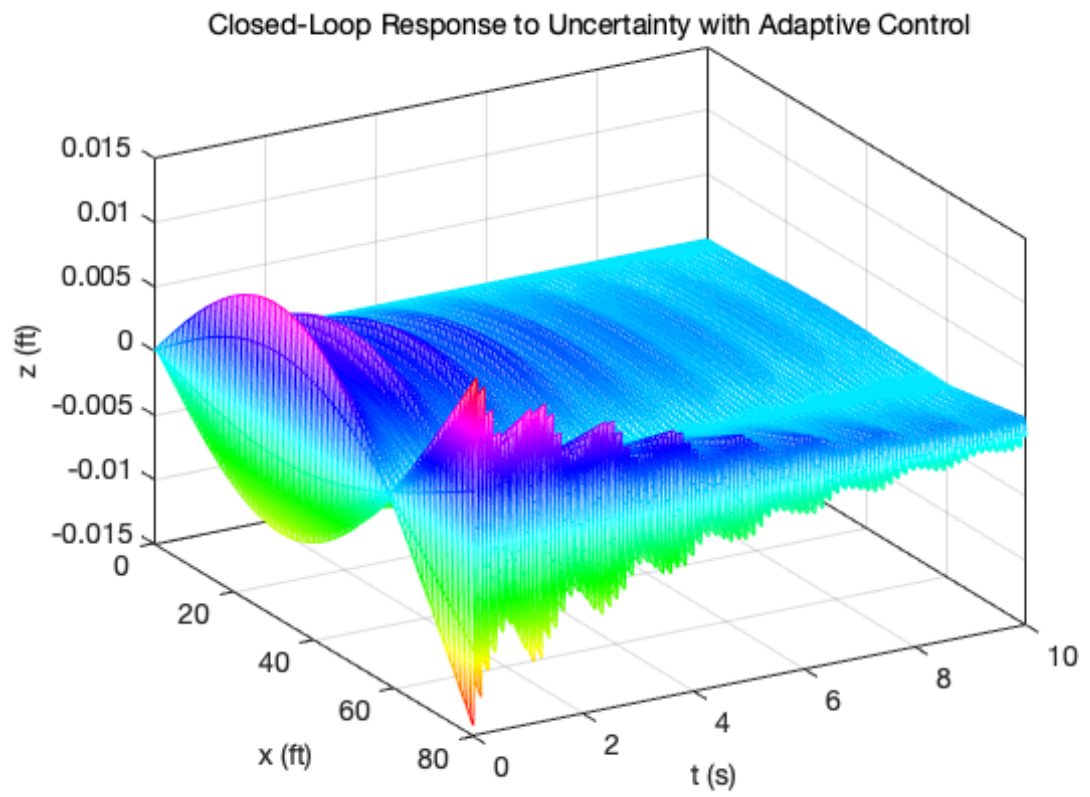

Figure 15. Wing Bending Deflection $z(x, t)$ with Adaptive Control 


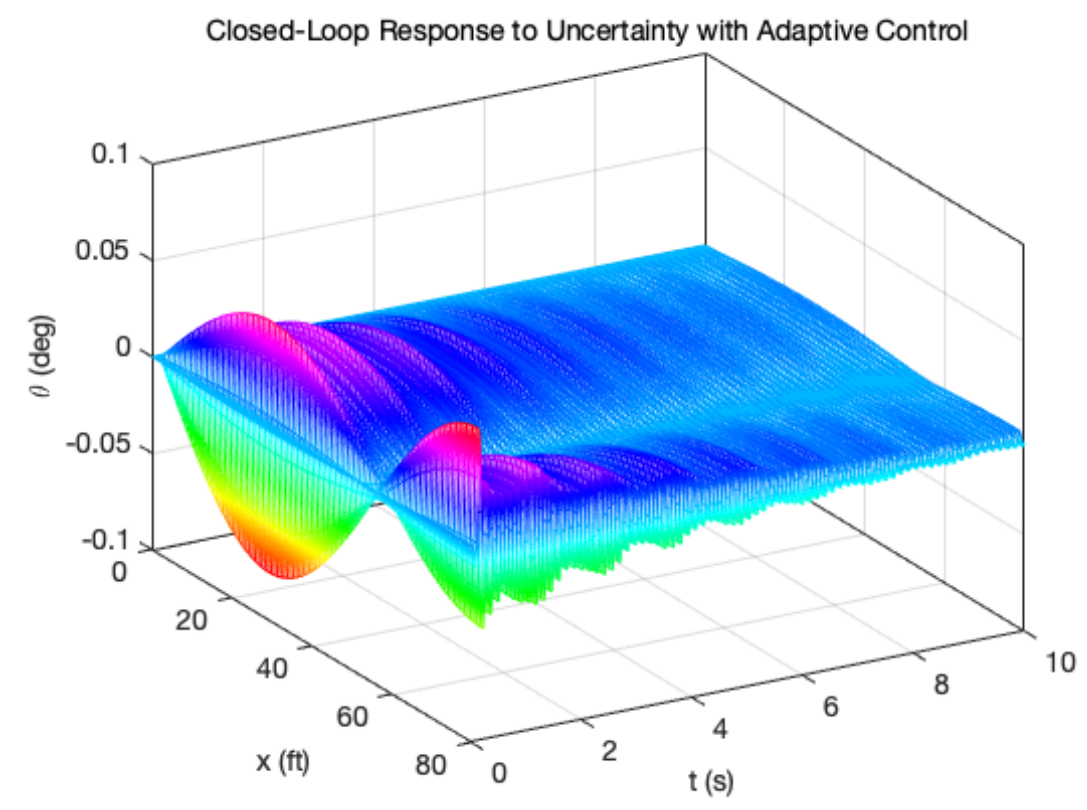

Figure 16. Wing Torsional Twist $\theta(x, t)$ with Adaptive Control

\section{Conclusion}

This paper presents a model-reference adaptive control theory for distributed Lagrangian infinite-dimensional systems using the Hamilton's principle. A generalized expression of the Euler-Lagrange equation is developed for a distributed system possessing a kinetic energy and a generalized potential energy of any arbitrary order. A linear operator is derived for this generalized potential energy. The Hamilton's principle in classical mechanics is applied to analyze the Hamiltonian function of the system which is a special case of the Lyapunov function. Two methods are developed for distributed control using the spatially distributed and continuous state information of the system. The first method is expressed in a strong form whereby no approximation is used. The distributed control synthesized by the strong-form method results in a nonlinear control. The second method is expressed in a weak form whereby one half of the distributed state information is approximated by a finite sum of orthogonal basis functions. This method results in a linear control. Model-reference adaptive control for this Lagrangian distributed system is developed using the weak-form control method.

The adaptive control theory is applied to a flutter suppression control of a flexible wing. Simulations show that the strong-form nominal controller produces less control effort than the weak-form nominal controller. In the presence of uncertainty, an adaptive augmentation control design is implemented for the weak-form nominal controller. The effectiveness of the adaptive augmentation is demonstrated by the simulation results.

\section{Acknowledgment}

The authors would like to acknowledge the funding support of this work by NASA Advanced Air Transport Technology project.

\section{References}

\footnotetext{
${ }^{1}$ Hughes, T. J. R., The Finite Element Method - Linear Static and Dynamic Finite Element Analysis, Prentice-Hall, Inc., 1987, pp. 3-44.

9 Bryson, A. E. and Ho, Y.-C., Applied Optimal Control Optimization, Estimation, and Control, Taylor \& Francis Group, New York, 1975.

6 Moore, B., "Principal Component Analysis in Linear Systems: Controllability, Observability, and Model Reduction," IEEE Tranactions on Automatic Control, Vol. AC-26, No. 1, February 1981.

7 Gawronski, W. K., Advanced Structural Dynamics and Active Control of Structures, Springer-Verlag, New York, 2004, pp. 65-107.
} 
5 Chan, P. H. M., Parker, A. R., Piazza, A., Bakalyar, J. A., Richards, W. L., "Real-Time Fiber Optics and Shape Sensing (FOSS) Technology," NASA Presentation, 29 February 2012, https://www.nasa.gov/pdf/625715main_3-5-C_Chan.pdf, Retrieval Date: December 1, 2019.

${ }^{8}$ Greenwood, D. T., Classical Dynamics, Dover Publications, Inc., New York, 1987.

2 Evans, L., Partial Differential Equations, American Mathematical Society, 2010.

3 Friedman, B., Principles and Techniques of Applied Mathematics, Dover Publication, Inc., New York, 1990.

4 Ioannou, P. and Kokotovic, P., "Instability Analysis and Improvement of Robustness of Adaptive Control," Automatica, Vol. 20, No. 5, 1984, pp. 583-594.

${ }^{11}$ Nguyen, N., "Optimal Control Modification for Robust Adaptive Control with Large Adaptive Gain," Systems \& Control Letters, 61 (2012) pp. 485-494, March 2012.

${ }^{12}$ Nguyen, N., Krishnakumar, K., and Boskovic, J., "An Optimal Control Modification to Model-Reference Adaptive Control for Fast Adaptation," AIAA Guidance, Navigation, and Control Conference, AIAA-2008-7283, August 2008.

${ }^{10}$ Nguyen, N., Ting, E., Nguyen, D., and Trinh, K., "Flutter Analysis of Mission-Adaptive Wing with Variable Camber Continuous Trailing Edge Flap," 55th AIAA/ASME/ASCE/AHS/ASC Structures, Structural Dynamics, and Materials Conference, AIAA-2014-0839, January 2014. 